\title{
Interferometric view of the circumstellar envelopes of northern FU Orionis-type stars
}

\author{
O. Fehér ${ }^{1,2}$, Á. Kóspál ${ }^{1,3}$, P. Ábrahám ${ }^{1}$, M. R. Hogerheijde ${ }^{4}$, and C. Brinch ${ }^{5}$ \\ 1 Konkoly Observatory, Research Centre for Astronomy and Earth Sciences, Hungarian Academy of Sciences, 1121 Budapest, \\ Konkoly Thege Miklós út 15-17, Hungary \\ e-mail: orsy.feher@gmail.com; feher.orsolya@csfk.mta.hu \\ 2 Eötvös Loránd University, Department of Astronomy, Pázmány Péter sétány 1/A, 1117 Budapest, Hungary \\ 3 Max-Planck-Institut für Astronomie, Königstuhl 17, 69117 Heidelberg, Germany \\ ${ }^{4}$ Leiden Observatory, Leiden University, Niels Bohrweg 2, 2333 CA Leiden, The Netherlands \\ 5 Niels Bohr International Academy, The Niels Bohr Institute, University of Copenhagen, Blegdamsvej 17, 2100 Copenhagen $\varnothing$, \\ Denmark
}

Received 26 June 2017 / Accepted 21 September 2017

\begin{abstract}
Context. FU Orionis-type objects are pre-main sequence, low-mass stars with large outbursts in visible light that last for several years or decades. They are thought to represent an evolutionary phase during the life of every young star when accretion from the circumstellar disk is enhanced during recurring time periods. These outbursts are able to rapidly build up the star while affecting the physical conditions inside the circumstellar disk and thus the ongoing or future planet formation. In many models, infall from a circumstellar envelope seems to be necessary to trigger the outbursts.

Aims. We characterise the morphology and the physical parameters of the circumstellar material around FU Orionis-type stars using the emission of millimetre-wavelength molecular tracers. The high-spatial-resolution study provides insight into the evolutionary state of the objects, the distribution of parameters in the envelopes and the physical processes forming the environment of these stars.

Methods. We observed the $J=1-0$ rotational transition of ${ }^{13} \mathrm{CO}$ and $\mathrm{C}^{18} \mathrm{O}$ towards eight northern FU Orionis-type stars (V1057 Cyg, V1515 Cyg, V2492 Cyg, V2493 Cyg, V1735 Cyg, V733 Cep, RNO 1B and RNO 1C) and determine the spatial and velocity structure of the circumstellar gas on a scale of a few thousand AU. We derive temperatures and envelope masses and discuss the kinematics of the circumstellar material.

Results. We detected extended CO emission associated with all our targets. Smaller-scale CO clumps were found to be associated with five objects with radii of 2000-5000 AU and masses of 0.02-0.5 $M_{\odot}$; these are clearly heated by the central stars. Three of these envelopes are also strongly detected in the $2.7 \mathrm{~mm}$ continuum. No central CO clumps were detected around V733 Cep and V710 Cas which can be interpreted as envelopes but there are many other clumps in their environments. Traces of outflow activity were observed towards V1735 Cyg, V733 Cep and V710 Cas.

Conclusions. The diversity of the observed envelopes enables us to set up an evolutionary sequence between the objects. We find their evolutionary state to range from early, embedded Class I stage to late, Class II-type objects with very-low-mass circumstellar material. We also find evidence of larger-scale circumstellar material influencing the detected spectral features in the environment of our targets. These results reinforce the idea of FU Orionis-type stars as representatives of a transitory stage between embedded Class I young stellar objects and classical T Tauri stars.
\end{abstract}

Key words. molecular data - stars: pre-main sequence - stars: variables: T Tauri, Herbig Ae/Be - circumstellar matter ISM: structure

\section{Introduction}

The class of low-mass pre-main sequence objects that show sudden, 5-6 mag brightness increase in the optical and nearinfrared (NIR) regimes was first described by Herbig (1977). These FU Orionis-type stars (FUors) remain bright for years or decades, sometimes fading slowly. The cause of the outburst is attributed to enhanced accretion (from a typical rate of $10^{-7}$ to $\left.10^{-4} M_{\odot} \mathrm{yr}^{-1}\right)$ from the circumstellar disk to the surface of the star (Hartmann \& Kenyon 1996). During one outburst up to $10^{-2} M_{\odot}$ mass can be accreted to the central star, playing a large role in accumulating the final stellar mass. The episodic FUor outbursts are predicted to change the parameters and structure of the circumstellar disk and envelope, for example, structural changes of the disk (Mosoni et al. 2013), signs of evaporation (Kun et al. 2011) or crystallisation (Ábrahám et al. 2009), the opening of the cavity (Ruíz-Rodríguez et al. 2017) and the movement of the snowline (Cieza et al. 2016). Theoretical explanations for the outburst phenomenon include thermal instabilities (Bell \& Lin 1994), gravitational instabilities (Armitage et al. 2001; Boley et al. 2006) and the perturbation of a close stellar or sub-stellar companion (Bonnell \& Bastien 1992). Nevertheless, continuous infall from a circumstellar envelope is needed to replenish the accreted material in the disk and certain studies show that this process might also play a role in triggering the outbursts (Vorobyov \& Basu 2006; Vorobyov et al. 2013).

In the optical regime FUors show similar spectra, with $F / G$ supergiant spectral types and broad absorption lines, while in the NIR they show K/M supergiant spectral types. The optical metallic lines (Fe I, Li I, Ca I) and the infrared CO absorption line are double-peaked and broadened, which is consistent 
Table 1. Observed targets and log of the observations.

\begin{tabular}{|c|c|c|c|c|c|c|c|c|c|}
\hline Target & other IDs & $\begin{array}{l}\text { RA (J2000) } \\
{[\mathrm{h}: \mathrm{m}: \mathrm{s}]}\end{array}$ & $\begin{array}{c}\operatorname{Dec}(\mathrm{J} 2000) \\
{\left[{ }^{\circ}::^{\prime \prime}\right]} \\
\end{array}$ & $\begin{array}{c}\mathrm{D} \\
{[\mathrm{pc}]}\end{array}$ & Telescope & Date & $\begin{array}{c}\text { Beam } \\
{[\operatorname{arcsec}]}\end{array}$ & $\begin{array}{l}\text { Merged beam } \\
\text { [arcsec] }\end{array}$ & $\begin{array}{c}\text { PA } \\
{[\mathrm{deg}]}\end{array}$ \\
\hline V1057 Cyg & HBC 300 & $20: 58: 53.73$ & $44: 15: 28.5$ & 600 & $\begin{array}{l}\text { PdBI } \\
30 \mathrm{~m}\end{array}$ & $\begin{array}{l}28 \text { March, } 2 \text { April } 2012 \\
\text { 19-22 June } 2012\end{array}$ & $\begin{array}{c}2.7 \times 2.2 \\
23.5\end{array}$ & $2.8 \times 2.3$ & 95 \\
\hline V1515 Cyg & HBC 692 & 20:23:48.02 & $42: 12: 25.8$ & 1000 & $\begin{array}{l}\text { PdBI } \\
30 \mathrm{~m}\end{array}$ & $\begin{array}{l}28 \text { March, } 2 \text { April } 2012 \\
\text { 20-22 June } 2012\end{array}$ & $\begin{array}{c}2.7 \times 2.2 \\
23.5\end{array}$ & $2.8 \times 2.3$ & 95 \\
\hline V2492 Cyg & PTF 10nvg & $20: 51: 26.23$ & $44: 05: 23.9$ & 550 & $\begin{array}{l}\mathrm{PdBI} \\
30 \mathrm{~m}\end{array}$ & $\begin{array}{l}28 \text { March, } 2 \text { April } 2012 \\
\text { 20-22 June } 2012\end{array}$ & $\begin{array}{c}2.8 \times 2.2 \\
23.5\end{array}$ & $2.8 \times 2.3$ & 95 \\
\hline V2493 Cyg & HBC 722 & 20:58:17.03 & $43: 53: 43.4$ & 550 & $\begin{array}{l}\text { PdBI } \\
30 \mathrm{~m}\end{array}$ & $\begin{array}{l}28 \text { March, } 2 \text { April } 2012 \\
\text { 20-22 June } 2012\end{array}$ & $\begin{array}{c}2.7 \times 2.2 \\
23.5\end{array}$ & $2.8 \times 2.3$ & 95 \\
\hline V1735 Cyg & Elias 1-12 & $21: 47: 20.66$ & 47:32:03.6 & 850 & $\begin{array}{l}\mathrm{PdBI} \\
30 \mathrm{~m}\end{array}$ & $\begin{array}{c}5 \text { Apr. } 2014 \\
\text { 25 June } 2014\end{array}$ & $\begin{array}{c}2.4 \times 2.2 \\
23.6\end{array}$ & $2.4 \times 2.2$ & 29.8 \\
\hline V733 Cep & Persson's Star & $22: 53: 33.26$ & $62: 32: 23.6$ & 900 & $\begin{array}{l}\mathrm{PdBI} \\
30 \mathrm{~m}\end{array}$ & $\begin{array}{l}\text { 4, } 18 \text { Apr. } 2014 \\
\text { 26 June } 2014\end{array}$ & $\begin{array}{c}2.4 \times 2.3 \\
23.6\end{array}$ & $2.5 \times 2.3$ & 43.6 \\
\hline V710 Cas & $\mathrm{RNO} 1 \mathrm{~B} / \mathrm{C}$ & 00:36:46.30 & $63: 28: 54.1$ & 800 & $\begin{array}{l}\mathrm{PdBI} \\
30 \mathrm{~m}\end{array}$ & $\begin{array}{c}\text { 6-7, } 17 \text { Apr. } 2014 \\
27 \text { June } 2014\end{array}$ & $\begin{array}{c}2.5 \times 2.2 \\
23.6\end{array}$ & $2.5 \times 2.4$ & 69.6 \\
\hline
\end{tabular}

Notes. The columns are: (1), (2) target name and alternative name; (3), (4) equatorial coordinates of the optical position of the target; (5) distance; (6) telescope; (7) observing dates; (8) single-dish or synthetic beam $H P B W$; (9) synthetic beam $H P B W$ of the merged observations; (10) position angle of the synthetic beam of the merged observations.

with a rotating disk origin. On the contrary, in the mid-infrared (MIR) they exhibit much higher variations, showing strong or weak silicate emission and even absorption. The far-infrared (FIR) and sub-millimetre continuum may also appear weaker or stronger in the different sources (Audard et al. 2014, and references therein). This diversity of the observed MIR and FIR FUor spectra is explained by Quanz et al. (2007b) proposing an evolutionary sequence that spans from younger FUors that are still embedded into dense, dusty envelopes, resembling Class I objects to more evolved stars showing many properties of Class II objects with a smaller, remnant envelope. Theoretical models (Vorobyov \& Basu 2015) predict that after a sequence of eruptions, the stars enter a more quiescent phase, becoming classical $\mathrm{T}$ Tauri stars. Thus, FUors might represent the link between Class I and Class II low-mass young stars. The outbursts are often accompanied by jets (Whelan et al. 2010), Herbig-Haro objects (Reipurth \& Wamsteker 1983; Bally \& Reipurth 2003), molecular outflows (Evans et al. 1994) and reflection nebulae (Goodrich 1987; Quanz et al. 2007b).

Millimetre-wavelength observations of molecular line and dust-continuum emission provide information about the smallscale structure and kinematics of the disk and the envelope around FUors. Low- and high-density circumstellar material can be mapped with transitions of ${ }^{12} \mathrm{CO},{ }^{13} \mathrm{CO}$ and $\mathrm{C}^{18} \mathrm{O}$ (Kóspál 2011; Hillenbrand et al. 2013; Kóspál et al. 2016, 2017), the outflows with ${ }^{12} \mathrm{CO}$ (Evans et al. 1994), the cavity walls with $\mathrm{HCO}^{+}$and $\mathrm{HCN}$ emission, while $\mathrm{SiO}$ and $\mathrm{SO}$ emission traces the shocked material inside the outflow (Hogerheijde et al. 1999). There are relatively few high-spatial-resolution studies of FUors (Momose et al. 1998; Hales et al. 2015; Kóspál et al. 2016; Zurlo et al. 2017; Ruíz-Rodríguez et al. 2017).

Here we present a ${ }^{13} \mathrm{CO}(1-0)$ and $\mathrm{C}^{18} \mathrm{O}(1-0)$ line survey with the aim of investigating the spatial and kinematic structure of the gas around eight FU Orionis-type young stars with a highspatial- and spectral-resolution interferometric survey. We calculate optical depths, excitation temperatures, column densities and continuum masses around the targets in Sect. 3, we describe the environment of the stars in detail and calculate line-based masses in Sect. 4, and we identify the envelopes, compare our study with previous results, and assess the evolutionary state of the observed FUors in Sect. 5.

\section{Observations and data reduction}

We observed seven northern FUor systems with the Plateau de Bure Interferometer (PdBI) and the IRAM $30 \mathrm{~m}$ telescope. The field centred on V710 Cas (RNO 1B) contains another close-by FUor, RNO $1 \mathrm{C}$, with a separation of $6^{\prime \prime}$. Table 1 lists the targets and the details of the observations.

V1057 Cyg, V1515 Cyg, V2492 Cyg and V2493 Cyg were observed with the PdBI on 28 March and on 2 April, 2012. The antennas were in the $6 \mathrm{Cq}$ configuration, providing $u v$ coverage between $15 \mathrm{~m}$ and $175 \mathrm{~m}$. The observations were done on the first night when the sources were setting and on the second night when they were rising, and each target was measured for about $10 \mathrm{~min}$, before switching to the next one, thus providing the best possible $u v$ coverage. The total on-source correlation time for each target was $2 \mathrm{~h}$. We used the $3 \mathrm{~mm}$ receiver centred on $109.0918 \mathrm{GHz}$, halfway between the ${ }^{13} \mathrm{CO}(1-0)$ and $\mathrm{C}^{18} \mathrm{O}(1-0)$ lines and each line was measured with a $20 \mathrm{MHz}$ wide correlator with $39 \mathrm{kHz}$ resolution. We also used two $160 \mathrm{MHz}$ wide correlators to measure the $2.7 \mathrm{~mm}$ continuum emission. The single dish half-power beam width (HPBW) at this wavelength is $45.8^{\prime \prime}$. Bright quasars were used for radio frequency bandpass, phase, and amplitude calibration and the flux scale was determined using the carbon star MWC 349. The weather conditions were stable throughout the observations, with precipitable water vapour between 5 and $9 \mathrm{~mm}$. The rms (root mean square) phase noise was typically below $30^{\circ}$ and the flux-calibration accuracy is estimated to be around $15 \%$. The single-dish observations were performed with the $30 \mathrm{~m}$ telescope on three nights between 20 and 22 June, 2012. We obtained Nyquist-sampled 112" $\times 112^{\prime \prime}$ on-the-fly maps with 7 " spacing using the Eight Mixer Receiver (EMIR) in frequency switching mode with $3.9 \mathrm{MHz}$ frequency throw in the $110 \mathrm{GHz}$ band with the Versatile Spectrometer Array (VESPA) that provided $20 \mathrm{MHz}$ bandwidth with $19 \mathrm{kHz}$ 
channel spacing. The ${ }^{13} \mathrm{CO}$ and $\mathrm{C}^{18} \mathrm{O}$ lines were covered in the UI sideband in this setup. The weather conditions were good, with precipitable water vapour between 2 and $8 \mathrm{~mm}$ on 28 March and between 2 and $4 \mathrm{~mm}$ on 2 April. V1735 Cyg, V733 Cep and V710 Cas were observed with the PdBI on 4-7 April and 17-18 April, 2014 and with the $30 \mathrm{~m}$ telescope on 25-27 June, 2014. Both the interferometric and the single-dish observations were made with the same instruments and settings as during the 2012 observing run. The weather conditions were good during both the single-dish and the interferometric observing sessions with precipitable water vapour of $1-15 \mathrm{~mm}$.

The data reduction of the single-dish spectra was done with the GILDAS-based CLASS and GREG ${ }^{1}$ software packages. We identified lines in the folded spectra, discarded those parts of the spectra where the negative signal from the frequency switching appeared, and subtracted a 4 th order polynomial baseline. The interferometric observations were reduced in the standard way with the GILDAS-based CLIC application, resulting in a primary beam of $45.8^{\prime \prime}$ and synthetic beams close to $2.5^{\prime \prime}$. For the line spectroscopy we merged the single-dish and interferometric measurements in the $u v$-space to correctly recover both the smaller- and larger-scale structure of the sources. First we took the Fourier transform of the single-dish images, then determined the shortest $u v$-distance in the interferometric dataset. A regular grid was made that filled out a circle within this shortest $u v$ distance, then we took the data points at these locations from the Fourier-transformed single-dish image and merged them with the original interferometric dataset. For the continuum, only the PdBI observations were combined. After this step, the imaging and cleaning was done in the usual manner.

\section{Results}

\subsection{Methods of CO line analysis}

The optical depth of the two isotopologues, $\tau_{13}$ and $\tau_{18}$, can be determined from the ratio of the ${ }^{13} \mathrm{CO}$ and $\mathrm{C}^{18} \mathrm{O}$ emission line peak main beam brightness on every pixel of the maps:

$\frac{T_{\mathrm{MB}}\left({ }^{13} \mathrm{CO}\right)}{T_{\mathrm{MB}}\left(\mathrm{C}^{18} \mathrm{O}\right)}=\frac{T_{\mathrm{ex},{ }^{13} \mathrm{CO}}\left(1-\mathrm{e}^{-\tau_{13}}\right)}{T_{\mathrm{ex}, \mathrm{C}^{18} \mathrm{O}}\left(1-\mathrm{e}^{-\tau_{18}}\right)}$,

where $T_{\mathrm{ex}}$ is the excitation temperature of the isotopologues. The ratio of the optical depths is given by the abundance ratio of the two isotopologues: $\tau_{13} / \tau_{18}=8$ (Wilson 1999). The excitation temperatures are assumed to be approximately equal.

From the radiative transfer equation we obtain

$I_{\mathrm{obs}}=\left(\mathrm{e}^{-\tau_{13}}-1\right) I_{\mathrm{bg}}+\left(1-\mathrm{e}^{-\tau_{13}}\right) B\left(T_{\mathrm{ex}}\right)$,

where $I_{\mathrm{obs}}$ is the observed intensity of the emission line and $I_{\mathrm{bg}}$ is the background intensity (corresponding to $T_{\mathrm{bg}}=2.7 \mathrm{~K}$ ). The contribution of $I_{\mathrm{bg}}$ is negligible compared to the line intensity, thus under local thermodynamic equilibrium (LTE) conditions and when the ${ }^{13} \mathrm{CO}$ emission is optically thick, the observed line intensity approaches the intensity emitted by a black-body with a temperature of $T_{\mathrm{ex}}$.

For deriving the column density the typically optically thin $\mathrm{C}^{18} \mathrm{O}$ line is the best choice. We use the method described by Scoville et al. (1986) to derive the column density of $\mathrm{C}^{18} \mathrm{O}$. If the populations of all energy levels of the molecule can be characterised by a single temperature $T_{\mathrm{ex}}$ derived from Eq. (2) and

\footnotetext{
1 http://wWw.iram.fr/IRAMFR/GILDAS
}

we assume that the vibrationally excited states are not populated and all energy levels are populated under LTE conditions, the total column density of the molecule is derived as

$N_{\mathrm{C}^{18} \mathrm{O}}=\frac{3 k_{\mathrm{B}}}{8 \pi^{3} B \mu^{2}} \frac{\mathrm{e}^{h B J(J+1) / k_{\mathrm{B}} T_{\mathrm{ex}}}}{(J+1)} \frac{T_{\mathrm{ex}}+h B / 3 k_{\mathrm{B}}}{1-\mathrm{e}^{-h v / k_{\mathrm{B}} T_{\mathrm{ex}}}} \int \tau_{18}(v) \mathrm{d} v$,

where $B$ and $\mu$ are the rotational constant and permanent dipole moment of the molecule, $k_{\mathrm{B}}$ is the Boltzmann-constant, $h$ is the Planck constant and $J$ is the rotational quantum number of the lower state in the observed transition. For the $J=1-0$ transition of $\mathrm{C}^{18} \mathrm{O}$ this gives

$N_{\mathrm{C}^{18} \mathrm{O}}=2.42 \times 10^{14} \int \frac{T_{\mathrm{ex}}+0.88}{1-\mathrm{e}^{-5.27 / T_{\mathrm{ex}}}} \tau_{18}(v) \mathrm{d} v$.

In the case of optically thin medium the total mass of $\mathrm{C}^{18} \mathrm{O}$ can be determined using

$M_{\mathrm{C}^{18} \mathrm{O}}=\frac{4 \pi d m_{\mathrm{C}^{18} \mathrm{O}} F_{\mathrm{ul}}}{h v_{\mathrm{ul}} A_{\mathrm{ul}} X_{\mathrm{u}}}$,

where $d$ is the distance of the source, $m_{\mathrm{C}^{18} \mathrm{O}}$ is the mass of the $\mathrm{C}^{18} \mathrm{O}$ molecule, $F_{\mathrm{ul}}$ is the observed integrated flux density, $v_{\mathrm{ul}}$ is the rest frequency of the transition between the $u$ upper level and 1 lower levels, $A_{\mathrm{ul}}$ is the Einstein-coefficient of the transition and $X_{\mathrm{u}}$ is the fractional population of the upper level (the ratio of the $\mathrm{C}^{18} \mathrm{O}(1-0)$ molecules to the total number of $\mathrm{C}^{18} \mathrm{O}$ molecules). In case of LTE, the level populations are thermalised and determined by the Boltzmann equation, thus $X_{u}$ can be computed if the temperature of the gas is known ( $T_{\text {ex }}$ from Eq. (2)). After deriving the $\mathrm{C}^{18} \mathrm{O}$ mass, the $M_{\mathrm{H}_{2}}$ hydrogen mass can be calculated using the canonical abundances of $\left[{ }^{12} \mathrm{CO}\right] /\left[\mathrm{C}^{18} \mathrm{O}\right]=560$ and $\left[\mathrm{H}_{2}\right] /\left[{ }^{12} \mathrm{CO}\right]=10^{4}$.

Due to the high signal-to-noise ratio $(\mathrm{S} / \mathrm{N})$ of the observations the formal uncertainty of the calculated masses are small, but there are several systematic factors affecting the results. The error in the target distances is around 10-20\% and the used temperature values can have around a factor of two uncertainties, since we assume homogeneous temperature distribution. In the case of masses derived from the continuum (see Sect. 3.2) the applied dust opacity coefficient may also have a factor of 2-4 uncertainty.

\subsection{Continuum}

The $2.7 \mathrm{~mm}$ continuum maps of the targets are shown in Fig. 1. The rms noise in the maps varies between $30-120 \mu \mathrm{Jy}_{\text {beam }}{ }^{-1}$. A single, roughly circular, compact source was detected at the optical position of V1057 Cyg with $70 \sigma$. The continuum emission of V2492 Cyg was detected with $79 \sigma$ with fainter emission extending to the southwest and another unresolved source with $16 \sigma$. We also detected V1735 Cyg with $38 \sigma$ and fainter emission extending around it to the north. Another $9 \sigma$ point source appears at $12^{\prime \prime}$ to the north. Four sources around V2493 Cyg and three bright sources around V710 Cas were observed but emission centred on the stars themselves was not detected. Only weak, $3 \sigma$ emission was detected close to the optical positions of V1515 Cyg and V733 Cep.

We derived the parameters of the sources by fitting twodimensional (2D) Gaussian functions using the software package CASA (McMullin et al. 2007). The resulting parameters are listed in Table 2 where the $3 \sigma$ upper limits of the peak fluxes of the undetected sources are given as well. V1057 Cyg and 
Table 2. Parameters of the detected continuum sources.

\begin{tabular}{|c|c|c|c|c|c|c|c|c|c|c|c|}
\hline Target & $\begin{array}{c}\text { RA } \\
{[\mathrm{h}: \mathrm{m}: \mathrm{s}]}\end{array}$ & $\begin{array}{c}\text { Dec } \\
{\left[:^{\circ}:^{\prime \prime}\right]}\end{array}$ & $\begin{array}{c}a_{\text {conv }} \\
{[\operatorname{arcsec}]}\end{array}$ & $\begin{array}{c}b_{\text {conv }} \\
{[\operatorname{arcsec}]}\end{array}$ & $\begin{array}{c}\mathrm{PA}_{\text {conv }} \\
{[\mathrm{deg}]}\end{array}$ & $\begin{array}{c}a_{\text {deconv }} \\
{[\operatorname{arcsec}]}\end{array}$ & $\begin{array}{c}b_{\text {deconv }} \\
{[\operatorname{arcsec}]}\end{array}$ & $\begin{array}{c}\mathrm{PA}_{\text {deconv }} \\
\text { [deg] }\end{array}$ & $\begin{array}{c}S_{v} \\
{[\mathrm{mJy}]}\end{array}$ & $\begin{array}{c}\mathrm{S}_{v, \mathrm{p}} \\
{\left[\mathrm{mJy}_{\text {beam }}{ }^{-1}\right]}\end{array}$ & $\begin{array}{l}M_{\text {cont }} \\
{\left[M_{\odot}\right]}\end{array}$ \\
\hline V1057 Cyg & 20:58:53.72 & $44: 15: 28.01$ & $3.4 \pm 0.1$ & $3.2 \pm 0.1$ & $176 \pm 37$ & $2.6 \pm 0.2$ & $1.8 \pm 0.3$ & $4 \pm 36$ & $4.9 \pm 0.2$ & $2.62 \pm 0.04$ & 0.39 \\
\hline V1515 Cyg & $20: 23: 47.95$ & $42: 12: 25.80$ & $\ldots$ & $\ldots$ & $\ldots$ & $\ldots$ & $\ldots$ & $\ldots$ & $\ldots$ & $0.18 \pm 0.03$ & 0.04 \\
\hline V2492 Cyg & $20: 51: 26.21$ & $44: 05: 23.83$ & $4.3 \pm 0.2$ & $2.7 \pm 0.1$ & $85 \pm 2$ & $3.3 \pm 0.2$ & $1.5 \pm 0.2$ & $82 \pm 3$ & $5.0 \pm 0.2$ & $2.65 \pm 0.04$ & 0.34 \\
\hline V2493 Cyg & $\ldots$ & $\ldots$ & $\ldots$ & $\ldots$ & $\ldots$ & $\ldots$ & $\ldots$ & $\ldots$ & $\ldots$ & $<0.24$ & $<0.02$ \\
\hline MMS1 & 20:58:16.52 & 43:53:53.34 & $3.0 \pm 0.1$ & $2.5 \pm 0.1$ & $98 \pm 5$ & $1.3 \pm 0.2$ & $1.1 \pm 0.3$ & $115 \pm 81$ & $4.5 \pm 0.2$ & $3.63 \pm 0.08$ & \\
\hline MMS2 & 20:58:16.79 & 43:53:35.61 & $2.9 \pm 0.2$ & $2.3 \pm 0.1$ & $99 \pm 9$ & $1.1 \pm 0.6$ & $0.6 \pm 0.4$ & $112 \pm 33$ & $1.6 \pm 0.2$ & $1.44 \pm 0.08$ & \\
\hline MMS3 & 20:58:17.66 & $43: 53: 30.20$ & $5.6 \pm 0.5$ & $3.2 \pm 0.2$ & $51 \pm 4$ & $5.0 \pm 0.5$ & $2.0 \pm 0.4$ & $47 \pm 5$ & $4.1 \pm 0.4$ & $1.37 \pm 0.08$ & \\
\hline MMS4 & $20: 58: 16.33$ & $43: 53: 25.97$ & $4.3 \pm 0.4$ & $3.1 \pm 0.2$ & $41 \pm 9$ & $3.6 \pm 0.6$ & $1.6 \pm 0.7$ & $34 \pm 12$ & $4.4 \pm 0.5$ & $2.02 \pm 0.08$ & \\
\hline V1735 Cyg & $21: 47: 20.65$ & $47: 32: 03.77$ & $2.9 \pm 0.2$ & $2.3 \pm 0.1$ & $19 \pm 8$ & $1.6 \pm 0.3$ & $0.9 \pm 0.4$ & $15 \pm 22$ & $2.3 \pm 0.2$ & $1.79 \pm 0.05$ & 0.37 \\
\hline V733 Cep & $22: 53: 33.47$ & $62: 32: 23.82$ & $\ldots$ & $\ldots$ & $\ldots$ & $\ldots$ & $\ldots$ & $\ldots$ & $\ldots$ & $0.38 \pm 0.10$ & 0.07 \\
\hline V710 Cas & $\ldots$ & $\ldots$ & $\ldots$ & $\ldots$ & $\ldots$ & $\ldots$ & $\ldots$ & $\ldots$ & $\ldots$ & $<0.36$ & $<0.05$ \\
\hline GM 1-33 & $00: 36: 47.36$ & $63: 29: 02.33$ & $2.6 \pm 0.2$ & $2.2 \pm 0.2$ & $14 \pm 18$ & $<1.6$ & $<0.9$ & $\ldots$ & $4.4 \pm 0.6$ & $4.14 \pm 0.12$ & \\
\hline \multirow[t]{2}{*}{ RNO 1D } & $00: 36: 46.66$ & $63: 28: 56.94$ & $4.8 \pm 0.6$ & $4.6 \pm 0.5$ & $21 \pm 82$ & $4.2 \pm 0.8$ & $4.0 \pm 0.8$ & $39 \pm 82$ & $9.4 \pm 1.3$ & $2.27 \pm 0.12$ & \\
\hline & $00: 36: 46.70$ & $63: 28: 49.47$ & $4.1 \pm 0.4$ & $3.9 \pm 0.4$ & $118 \pm 78$ & $3.5 \pm 0.7$ & $3.0 \pm 0.6$ & $106 \pm 86$ & $9.0 \pm 1.2$ & $2.97 \pm 0.12$ & \\
\hline
\end{tabular}

Notes. The columns are: (1) target name; (2), (3) equatorial coordinates of the continuum source; (4)-(6) major, minor axis and position angle of the fitted 2D Gaussian (convolved with beam); (7)-(9) major axis, minor axis, and position angle of fitted 2D Gaussian (deconvolved); (10) integrated flux; (11) peak flux; (12) continuum mass.

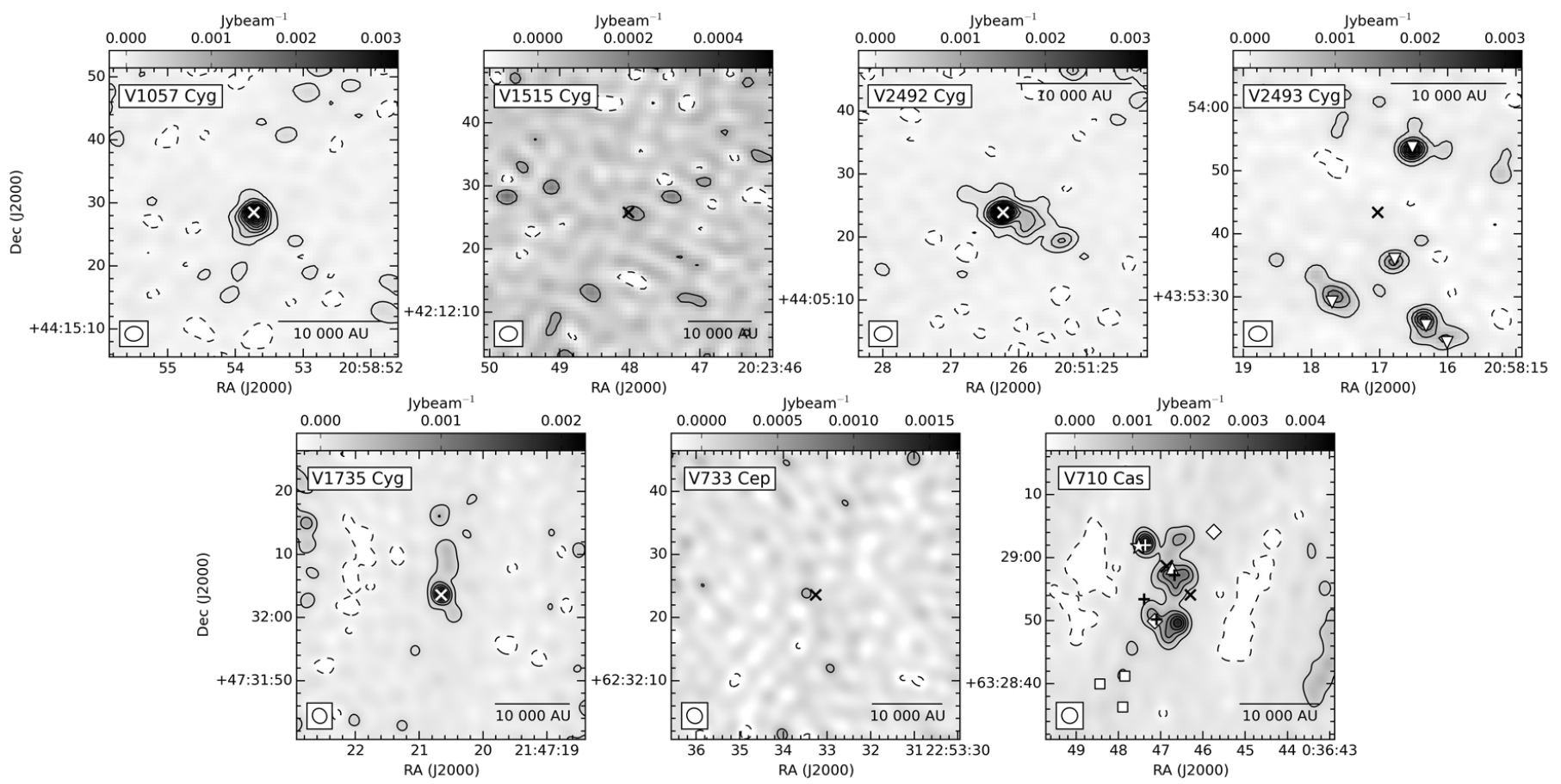

Fig. 1. $2.7 \mathrm{~mm}$ continuum maps of the sources. No short-spacing data was combined with the interferometric observations of the continuum. The solid lines mark the $3 \sigma, 9 \sigma, 15 \sigma \ldots$ contour levels and dashed lines show the $-3 \sigma$ contour level. Crosses mark the positions of the FUors and the beam is shown in the bottom-left corner. Other associated sources are marked as follows: upside down triangles mark the millimetre sources around V2493 Cyg from Dunham et al. (2012), in the region around V710 Cas (RNO 1B) a second cross marks RNO 1C, an asterisk marks IRAS 00338+6312 (Staude \& Neckel 1991), crosses mark the radio sources from Anglada et al. (1994), triangle marks the sub-millimetre source from Sandell \& Weintraub (2001), squares mark the IRAC sources from Quanz et al. (2007a) and diamonds mark RNO 1F and RNO 1G.

V2492 Cyg are resolved, while V1735 Cyg is only marginally resolved in one direction with $b_{\text {deconv }}=0.9 \pm 0.4^{\prime \prime}$. The rest of the continuum sources are resolved, except MMS2, located close to V2493 Cyg. The detected FUors have deconvolved sizes between 500 and $1800 \mathrm{AU}$. The measured size and integrated flux of V2492 Cyg is close to the one derived by Hillenbrand et al. (2013) who gave a deconvolved size of $2.7^{\prime \prime} \times 1.5^{\prime \prime}$ with a position angle of $66^{\circ}$ and an integrated flux of $5.6 \pm 0.26 \mathrm{Jy}$ for their source. Kóspál et al. (2016) give a detailed description of the observed continuum sources around V2493 Cyg. Both our line and continuum observations for V2493 Cyg were partly published there and is only briefly discussed here.

The mass of the continuum sources was derived using the equation

$M_{\mathrm{cont}}=\frac{g S_{v} d^{2}}{\kappa_{v} B_{v}(T)}$ 

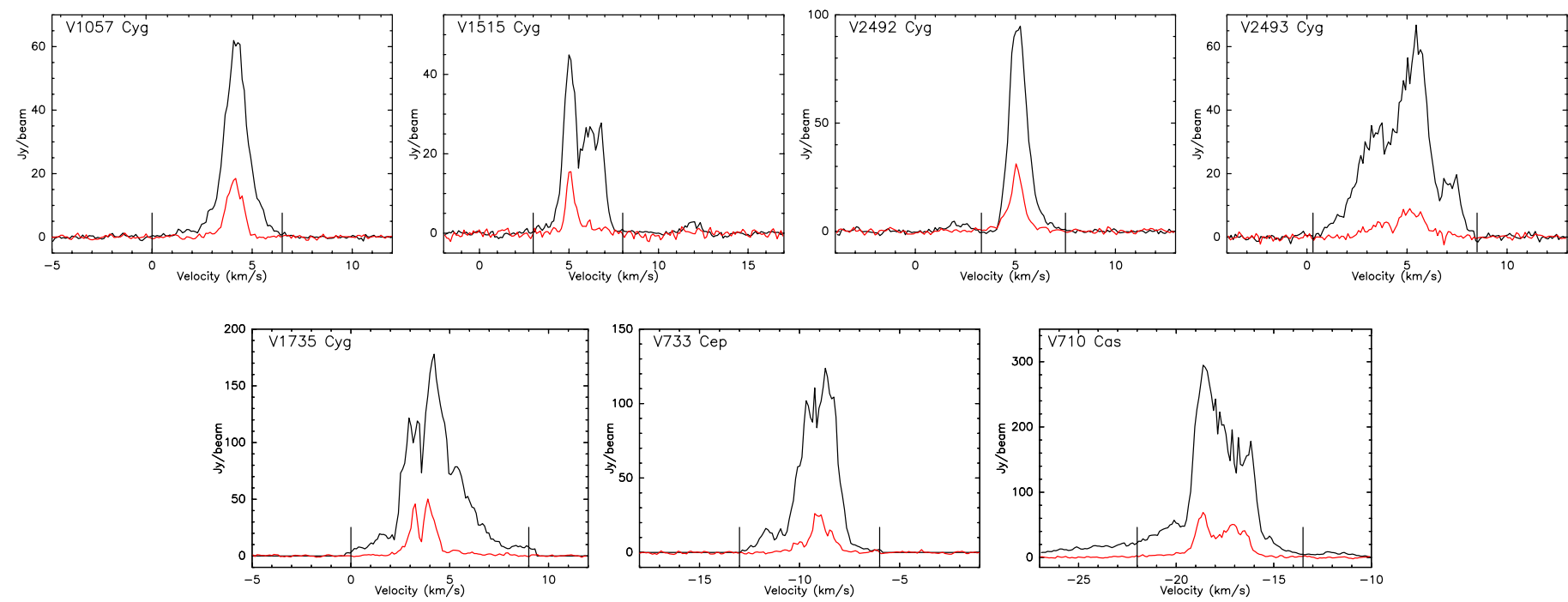

Fig. 2. ${ }^{13} \mathrm{CO}(1-0)$ (black) and $\mathrm{C}^{18} \mathrm{O}(1-0)$ (red) spectra integrated over the beam size $\left(\approx 2.5^{\prime \prime}\right)$ around the centre position of our maps. Vertical lines mark the velocity range where the moments shown in Figs. 3-10 were derived.

Table 3. Parameters of the spectra at the centre of each map averaged over one beam.

\begin{tabular}{ccccccr}
\hline \hline Target & $\begin{array}{c}v_{\mathrm{LSR}}\left({ }^{13} \mathrm{CO}\right) \\
{\left[\mathrm{km} \mathrm{s}^{-1}\right]}\end{array}$ & $\begin{array}{c}\Delta v\left({ }^{13} \mathrm{CO}\right) \\
{\left[\mathrm{km} \mathrm{s}^{-1}\right]}\end{array}$ & $\begin{array}{c}\mathrm{S}_{\mathrm{p}}\left({ }^{13} \mathrm{CO}\right) \\
{\left[\mathrm{mJy} \mathrm{beam}^{-1}\right]}\end{array}$ & $\begin{array}{c}v_{\mathrm{LSR}}\left(\mathrm{C}^{18} \mathrm{O}\right) \\
{\left[\mathrm{km} \mathrm{s}^{-1}\right]}\end{array}$ & $\begin{array}{c}\Delta v\left(\mathrm{C}^{18} \mathrm{O}\right) \\
{\left[\mathrm{km} \mathrm{s}^{-1}\right]}\end{array}$ & $\begin{array}{r}\mathrm{S}_{\mathrm{p}}\left(\mathrm{C}^{18} \mathrm{O}\right) \\
{\left[\mathrm{mJy} \mathrm{beam}^{-1}\right]}\end{array}$ \\
\hline V1057 Cyg & 4.05 & 1.97 & $400 \pm 1$ & 4.10 & 1.18 & $79 \pm 1$ \\
V1515 Cyg & 5.80 & 1.97 & $397 \pm 2$ & 5.38 & 1.66 & $147 \pm 2$ \\
V2492 Cyg & 4.97 & 1.17 & $480 \pm 2$ & 4.90 & 1.00 & $117 \pm 1$ \\
V2493 Cyg & 4.65 & 3.44 & $507 \pm 1$ & 4.79 & 2.50 & $75 \pm 1$ \\
V1735 Cyg & 4.16 & 3.87 & $418 \pm 1$ & 3.89 & 2.63 & $139 \pm 2$ \\
V733 Cep & -9.23 & 2.50 & $424 \pm 2$ & -8.99 & 1.65 & $97 \pm 2$ \\
V710 Cas & -17.83 & 3.38 & $943 \pm 8$ & -17.72 & 2.87 & $163 \pm 2$ \\
\hline
\end{tabular}

Notes. The columns are: (1) target name; (2) intensity averaged velocity of the ${ }^{13} \mathrm{CO}$ line (first order moment); (3) ${ }^{13} \mathrm{CO}$ linewidth (from the second order moment); (4) peak flux density of the ${ }^{13} \mathrm{CO}$ line; (5)-(7) the same parameters for the $\mathrm{C}^{18} \mathrm{O}$ line.

where $g=100$ is the gas-to-dust ratio, $S_{v}$ is the measured flux density at $2.7 \mathrm{~mm}, d$ is the distance, $\kappa_{v}=0.2 \mathrm{~cm}^{2} \mathrm{~g}^{-1}$ is the dust opacity coefficient at $2.7 \mathrm{~mm}$ based on Ossenkopf \& Henning (1994) and $B_{v}(T)$ is the Planck function for a black-body with a temperature of $T$. The derived masses of the continuum sources coinciding with the targeted FUors using $T=30 \mathrm{~K}$ are in Table 2.

\section{3. ${ }^{13} \mathrm{CO}(1-0)$ and $\mathrm{C}^{18} \mathrm{O}(1-0)$}

Both ${ }^{13} \mathrm{CO}$ and $\mathrm{C}^{18} \mathrm{O}$ emission were detected at each target with high $\mathrm{S} / \mathrm{N}$. The averaged spectra integrated over one synthetic beam at the position of the FUors (the centre of the maps) are shown in Fig. 2 and the parameters of these spectra are listed in Table 3. The channel maps are shown in Figs. A.1-A.14. The rms noise per channel ranges from $\sigma=0.008-0.04 \mathrm{Jy}_{\text {beam }}{ }^{-1}$.

The ${ }^{13} \mathrm{CO}$ line has a symmetric, Gaussian-like shape towards V1057 Cyg and V2492 Cyg, while it seems to be somewhat self-absorbed towards V733 Cep. The spectra taken towards the other sources show multiple peaks that are either caused by selfabsorption or might correspond to different line components in the line of sight, blended together. These blended line components also appear in the $\mathrm{C}^{18} \mathrm{O}$ spectra. We find the broadest ${ }^{13} \mathrm{CO}$ lines at the position of V710 Cas with $\Delta v \approx 3.8 \mathrm{~km} \mathrm{~s}^{-1}$ and the smallest ${ }^{13} \mathrm{CO}$ linewidth at V2492 Cyg with $\Delta v \approx 1.2 \mathrm{~km} \mathrm{~s}^{-1}$. Apart from the bright, main ${ }^{13} \mathrm{CO}$ line component we detected another velocity components in the direction of V1515 Cyg and V2492 Cyg, at $11.9 \mathrm{~km} \mathrm{~s}^{-1}$ and at $2.1 \mathrm{~km} \mathrm{~s}^{-1}$, respectively. These line components are not well visible in Fig. 2, because they mainly originate from a different region, not the central beam.

The integrated intensity (zeroth order moment) and the intensity weighted average velocity (first order moment) maps towards each target are plotted in panels a-d in Figs. 3-10. The ${ }^{13} \mathrm{CO}$ and $\mathrm{C}^{18} \mathrm{O}$ integrated intensity peaks are generally close to the optical stellar positions but considering the pointing accuracy of $\approx 0.5^{\prime \prime}$ of the observations, only the optical position of V1735 Cyg coincides with the detected ${ }^{13} \mathrm{CO}$ peak and the optical position of V2492 Cyg coincides with the $\mathrm{C}^{18} \mathrm{O}$ peak. The pointing accuracy was calculated from the beam size, the $\mathrm{S} / \mathrm{N}$ and the absolute positional accuracy of the telescope. V1057 Cyg and V1735 Cyg show a morphology with one strong, distinct integrated intensity peak at the centre, but many smaller local peaks or clumps are found in the region around other targets, for example, V1515 Cyg or V2493 Cyg, while V733 Cep and V710 Cas do not seem to coincide with $\mathrm{CO}$ emission peaks.

Using Eq. (1) to calculate optical depth, the ${ }^{13} \mathrm{CO}$ emission was found mostly optically thick around the targets with values up to 15 , but smaller areas with $\tau_{13}$ lower than unity can also 

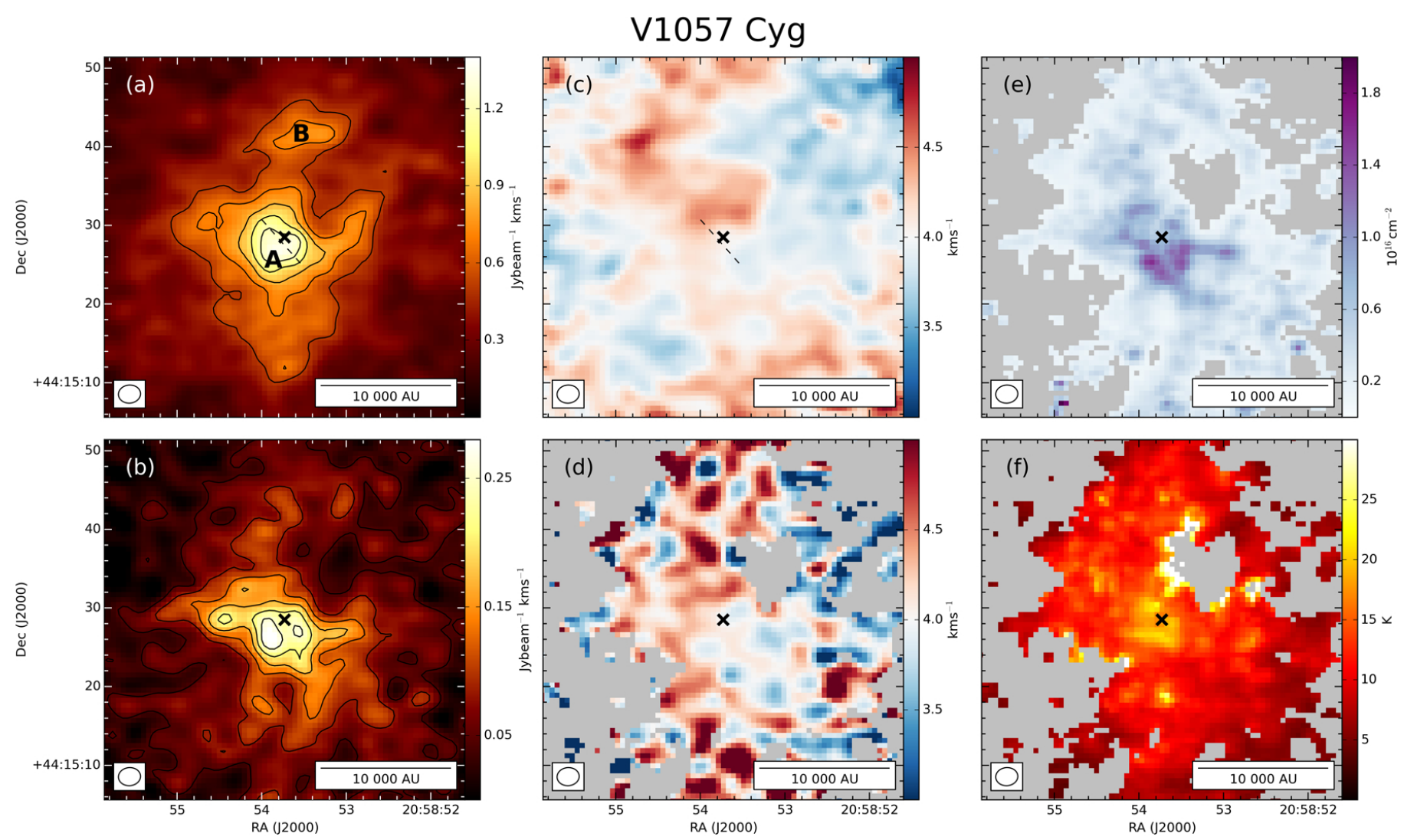

Fig. 3. Moment and physical parameter maps of V1057 Cyg: $a){ }^{13} \mathrm{CO}$ integrated intensity; $\left.b\right) \mathrm{C}^{18} \mathrm{O}$ integrated intensity; $\left.c\right){ }^{13} \mathrm{CO}$ intensity weighted velocity; d) $\mathrm{C}^{18} \mathrm{O}$ intensity weighted velocity; e) $\mathrm{C}^{18} \mathrm{O}$ column density; $f$ ) temperature. The black cross marks the position of the FUor and letters indicate the clumps that are discussed in the text. The solid contours mark the $n \sigma_{\text {int }}$ levels (the multiples of the noise level on the map); $\sigma_{\text {int }}=0.17 \mathrm{Jy} \mathrm{beam}^{-1} \mathrm{~km} \mathrm{~s}^{-1}$ and $n=3,4, \ldots, 8$ on $(a)$ and $\sigma_{\text {int }}=0.014 \mathrm{Jy} \mathrm{beam}^{-1} \mathrm{~km} \mathrm{~s}^{-1}$ and $n=3,6, \ldots, 18$ on $(b)$. The pixels with $\mathrm{C}^{18} \mathrm{O}$ peak values less than $9 \sigma$ are coloured grey on panels $d, e$ and $f$.

be observed. The $\mathrm{C}^{18} \mathrm{O}$ emission is generally optically thin with regions around V1515 Cyg and V733 Cep having $\tau_{18}$ somewhat higher than unity. The temperature distribution around the objects, as calculated from Eq. (2), generally peaks close to the position of the FUors, for example, at V1057 Cyg or V1515 Cyg, but there are sources with complicated temperature distributions, for example, V2493 Cyg or V710 Cas. The peak temperatures are between $15-75 \mathrm{~K}$ with the less dense material showing $5-25 \mathrm{~K}$. The $\mathrm{C}^{18} \mathrm{O}$ column densities are above $10^{14} \mathrm{~cm}^{-2}$ around all our targets, corresponding to $\mathrm{H}_{2}$ column densities of higher than $5.6 \times 10^{20} \mathrm{~cm}^{-2}$, using the previously cited abundancies. The highest $\mathrm{C}^{18} \mathrm{O}$ column density peaks appear at V710 Cas with $6-7 \times 10^{16} \mathrm{~cm}^{-2}$ and the lowest is at V1515 Cyg and V733 Cep with $7 \times 10^{15} \mathrm{~cm}^{-2}$. The $\mathrm{C}^{18} \mathrm{O}$ column density and the temperature maps are plotted in panels (e) and (f) in Figs. 3-10. In the regions where the $\mathrm{C}^{18} \mathrm{O}$ opacities are approaching zero or there is higher noise in the $\mathrm{C}^{18} \mathrm{O}$ spectra, undefined optical depths, temperatures and densities appear; these regions are masked out and plotted with grey in the Figures.

Previous interferometric observations of V1057 Cyg, V1515 Cyg and V1735 Cyg were presented by Kóspál (2011). Those ${ }^{13} \mathrm{CO}(1-0)$ observations were made with the PdBI in 1993, using four antennas in the 4D1 configuration with baselines ranging from 24 to $64 \mathrm{~m}$. The synthesised beam was approximately $7^{\prime \prime} \times 6^{\prime \prime}$ and the rms noise per channel was around $0.15 \mathrm{Jy}$ beam $^{-1}$. The $2.7 \mathrm{~mm}$ continuum was also measured towards the targets. V1057 Cyg and V1515 Cyg were not detected in those continuum measurements, while here we did detect both. They observed somewhat different morphologies around the targets but the discrepancies originate from the lower spatial resolution, the higher noise level and the sparser $u v$-coverage of the 1993 observations; further discussed below.

\section{Analysis of individual objects}

\subsection{V1057 Cyg}

The ${ }^{13} \mathrm{CO}$ and $\mathrm{C}^{18} \mathrm{O}$ lines towards the FUor are single-peaked, without significant self-absorption. Some excess emission on the ${ }^{13} \mathrm{CO}$ blue line wing can be detected between 0 and $3.4 \mathrm{~km} \mathrm{~s}^{-1}$. The integrated intensity map of ${ }^{13} \mathrm{CO}$ shows one strong peak at $1.5^{\prime \prime}$ to the south from the centre with $1.3 \mathrm{Jy} \mathrm{beam}^{-1} \mathrm{~km} \mathrm{~s}^{-1}$ (see Fig. 3a). The central clump around this peak (clump A) is roughly circular, with a radius of $5^{\prime \prime}$, and it appears in $\mathrm{C}^{18} \mathrm{O}$ as well. It also roughly corresponds to the detected continuum source but it is centred more to the southeast. Two protrusions to the east and to the west from clump A can be observed in the integrated intensity of both $\mathrm{CO}$ isotopologues. The western protrusion extends towards the nebulosity "arm" seen on optical images (Duncan et al. 1981). Integrating the excess ${ }^{13} \mathrm{CO}$ emission between $0-3.4 \mathrm{~km} \mathrm{~s}^{-1}$ also shows this western protrusion and additionally, clump B. A ${ }^{13} \mathrm{CO}$ and $\mathrm{C}^{18} \mathrm{O} v_{\text {LSR }}$ gradient can be observed in clump A in the southwest-northeast direction (Fig. 3c), which can be seen on the channel maps as well (Figs. A.1 and A.2) where clump A appears at around $3.33 \mathrm{~km} \mathrm{~s}^{-1}$ to the south from the centre then moves towards the east between 4.17 and $4.49 \mathrm{~km} \mathrm{~s}^{-1}$. The ${ }^{13} \mathrm{CO}$ velocities along the line marked in 


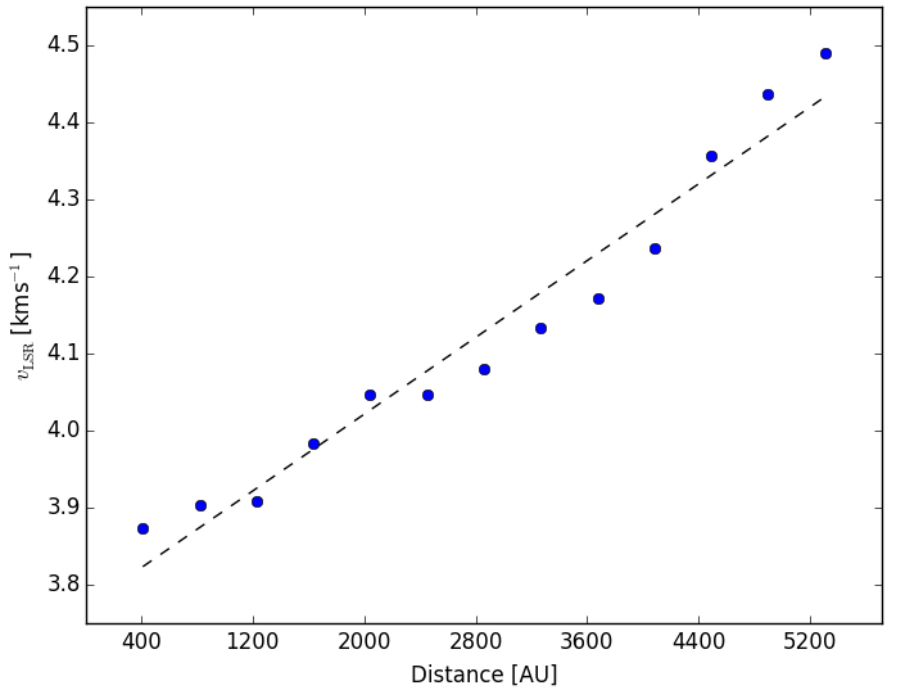

Fig. 4. ${ }^{13} \mathrm{CO}(1-0) v_{\text {LSR }}$ gradient across clump A of V1057 Cyg along the line marked on panels a and $\mathrm{c}$ in Fig. 3. The dashed line shows a linear fit to the values.

panels a and c in Fig. 3 are plotted and fitted with a line in Fig. 4. The velocity gradient is $0.08 \mathrm{~km} \mathrm{~s}^{-1} \operatorname{arcsec}^{-1}$ or $27.6 \mathrm{~km} \mathrm{~s}^{-1} \mathrm{pc}^{-1}$ at a distance of $600 \mathrm{pc}$. It is also apparent that at lower velocities the more diffuse emission in the channel maps is elongated to the west-east direction but it becomes elongated in the north-south direction at higher velocities, where clump $\mathrm{B}$ also merges with the central region. The $\mathrm{C}^{18} \mathrm{O}$ channel maps (Fig. A.2) are very similar to the ${ }^{13} \mathrm{CO}$ at corresponding velocities, which suggests that the emission of the two molecules originate in the same volume of material.

The calculated temperatures are higher in clump A $(20-22 \mathrm{~K})$ than in the more diffuse region outside of it. This suggests heating from the central source, although there is a local minimum at the position of the FUor with $17 \mathrm{~K}$ inside clump A. The ${ }^{13} \mathrm{CO}$ emission is only marginally optically thick inside clump A with $\tau_{13}=1-3$. The $\mathrm{C}^{18} \mathrm{O}$ column density is above $10^{16} \mathrm{~cm}^{-2}$ in the southern part of clump A with a peak of $1.6 \times 10^{16} \mathrm{~cm}^{-2}$ and there are at least three arcs of denser material around this region. In the northern half of clump A however, the density decreases to $4.5 \times 10^{15} \mathrm{~cm}^{-2}$. The mass of clump A using an average $T=16.4 \mathrm{~K}$ is $0.21 M_{\odot}$, which is almost twice the value derived by Kóspál (2011) from ${ }^{13} \mathrm{CO}$ emission. Assuming that the ${ }^{13} \mathrm{CO}$ emission is optically thick and deriving the mass from it we get $0.14 M_{\odot}$, which is more consistent with their result, implying that the difference originates from ${ }^{13} \mathrm{CO}$ being optically thick. The temperature peak measured close to the FUor and the detected velocity gradient might signal a rotating envelope with a radius of $5^{\prime \prime}$ (3000 AU).

\subsection{V1515 Cyg}

The ${ }^{13} \mathrm{CO}$ line of V1515 Cyg shows two peaks with possible self absorption in the mapped area with no significant excess on the line wings. The brightest peak of the $\mathrm{C}^{18} \mathrm{O}$ line is at the same velocity as the ${ }^{13} \mathrm{CO}$ peak and traces of another line component also appear. Additionally, apart from this bright line at $5.7 \mathrm{~km} \mathrm{~s}^{-1}$, there is another, faint ${ }^{13} \mathrm{CO}$ velocity component at around $11.8 \mathrm{~km} \mathrm{~s}^{-1}$. Its integrated intensity shows a flaring structure that covers the northwest region of the map. The integrated intensity map of the brighter line shows a clumpy, extended, roughly spherical structure (Fig. 5a) that fills the primary beam. The maximum is inside clump A, with $1.1 \mathrm{Jy} \mathrm{beam}^{-1} \mathrm{~km} \mathrm{~s}^{-1}$, roughly coinciding with the FUor. Three other clumps (B, C and D) are seen at 9 and $13.5^{\prime \prime}$ to the northwest and at $8.7^{\prime \prime}$ to the west. A hole in the emission appears between clump A and C. The overall distribution of stronger and weaker peaks actually correspond well to the arc-like shape detected by Kóspál (2011) considering the lower resolution of their measurement. This arclike shape is oriented similarly as the arc of nebulosity on optical images. No clear velocity gradient can be defined.

Looking at the ${ }^{13} \mathrm{CO}$ channel maps (Fig. A.3) first we see clump A appearing at low velocities, then gradually many smaller clumps emerge around it in a ring. These clumps fragment, merge and change positions slightly throughout the measured velocity interval. Clump A disappears around $5.63 \mathrm{~km} \mathrm{~s}^{-1}$ but some of the smaller clumps are visible at even $5.9 \mathrm{~km} \mathrm{~s}^{-1}$. Above $6 \mathrm{~km} \mathrm{~s}^{-1}$ only diffuse, extended emission with small local peaks is present. This morphology may suggest an expanding bubble around the star, where at low velocities we detect the approaching wall of the structure, at medium velocities the ringlike circumference, then at even higher velocities the far side of the bubble wall moving away from the observer. If the structure is indeed expanding, the velocity differences and the size of the ring would suggest a dynamical age of $\approx 32000 \mathrm{yr}$.

The $\mathrm{C}^{18} \mathrm{O}$ integrated intensity map is somewhat different from the ${ }^{13} \mathrm{CO}$ emission, showing an elongated structure in the northwest-southeast direction. Three strong peaks (around $0.2 \mathrm{Jy}$ beam $^{-1} \mathrm{~km} \mathrm{~s}^{-1}$ ) can be seen at $7^{\prime \prime}$ to the southeast, at $11^{\prime \prime}$ to the northwest, and close to the centre. Similarly in the $\mathrm{C}^{18} \mathrm{O}$ channel maps (Fig. A.4), there are only 3-4 larger clumps present between 5.1 and $5.32 \mathrm{~km} \mathrm{~s}^{-1}$, two of these approximately coincide with clumps $\mathrm{A}$ and $\mathrm{C}$. There are another 3-4 clumps at the western edge of the map between 5.53 and $5.85 \mathrm{~km} \mathrm{~s}^{-1}$, where the ${ }^{13} \mathrm{CO}$ emission is much less significant at these velocities.

The high-density regions in Fig. 5e coincide with the positions of the clumps that appear both in some of the ${ }^{13} \mathrm{CO}$ and $\mathrm{C}^{18} \mathrm{O}$ channel maps. In these clumps the $\mathrm{C}^{18} \mathrm{O}$ emission becomes optically thick. The $N_{\mathrm{C}^{18} \mathrm{O}}$ distribution peaks in clump $\mathrm{C}$ with $5 \times 10^{15} \mathrm{~cm}^{-2}$ and in the other clumps with $\approx 4.5 \times 10^{15} \mathrm{~cm}^{-2}$. The temperature distribution peaks at the centre in clump A with $14.7 \mathrm{~K}$, while most of the mapped area has temperatures around $8-10 \mathrm{~K}$. The mass of clump A with a radius of $5^{\prime \prime}$ and an average temperature of $11.2 \mathrm{~K}$ is $0.26 M_{\odot}$ from $\mathrm{C}^{18} \mathrm{O}$ flux density integrated between 4 and $5.53 \mathrm{~km} \mathrm{~s}^{-1}$, where clump $\mathrm{A}$ is present in the channel maps. This is lower than the value of $0.42 M_{\odot}$ derived by Kóspál (2011) using ${ }^{13} \mathrm{CO}$, which again may originate from ${ }^{13} \mathrm{CO}$ optical depth effects. We note however that the previous observations did not resolve the structure around V1515 Cyg into clumps. The three larger clumps in the $\tau_{13}$ ring have average temperatures of $9-11 \mathrm{~K}$ and masses of $0.04-0.05 M_{\odot}$.

\subsection{V2492 Cyg}

The ${ }^{13} \mathrm{CO}$ line in V2492 Cyg is single-peaked and shows no significant self-absorption or line wing excesses. Apart from the brighter line around $5 \mathrm{~km} \mathrm{~s}^{-1}$, there is another faint ${ }^{13} \mathrm{CO}$ velocity component at $2 \mathrm{~km} \mathrm{~s}^{-1}$. The integrated intensity of this component shows emission at the centre and in a point sourcelike peak inside diffuse emission at $22^{\prime \prime}$ to the northeast from the centre. The integrated intensity map of the brighter ${ }^{13} \mathrm{CO}$ line component shows an asymmetric, north-south oriented morphology with emission missing to the northeast, east and southeast (Fig. 6a). The primary peak is at $1.3^{\prime \prime}$ to the west from 

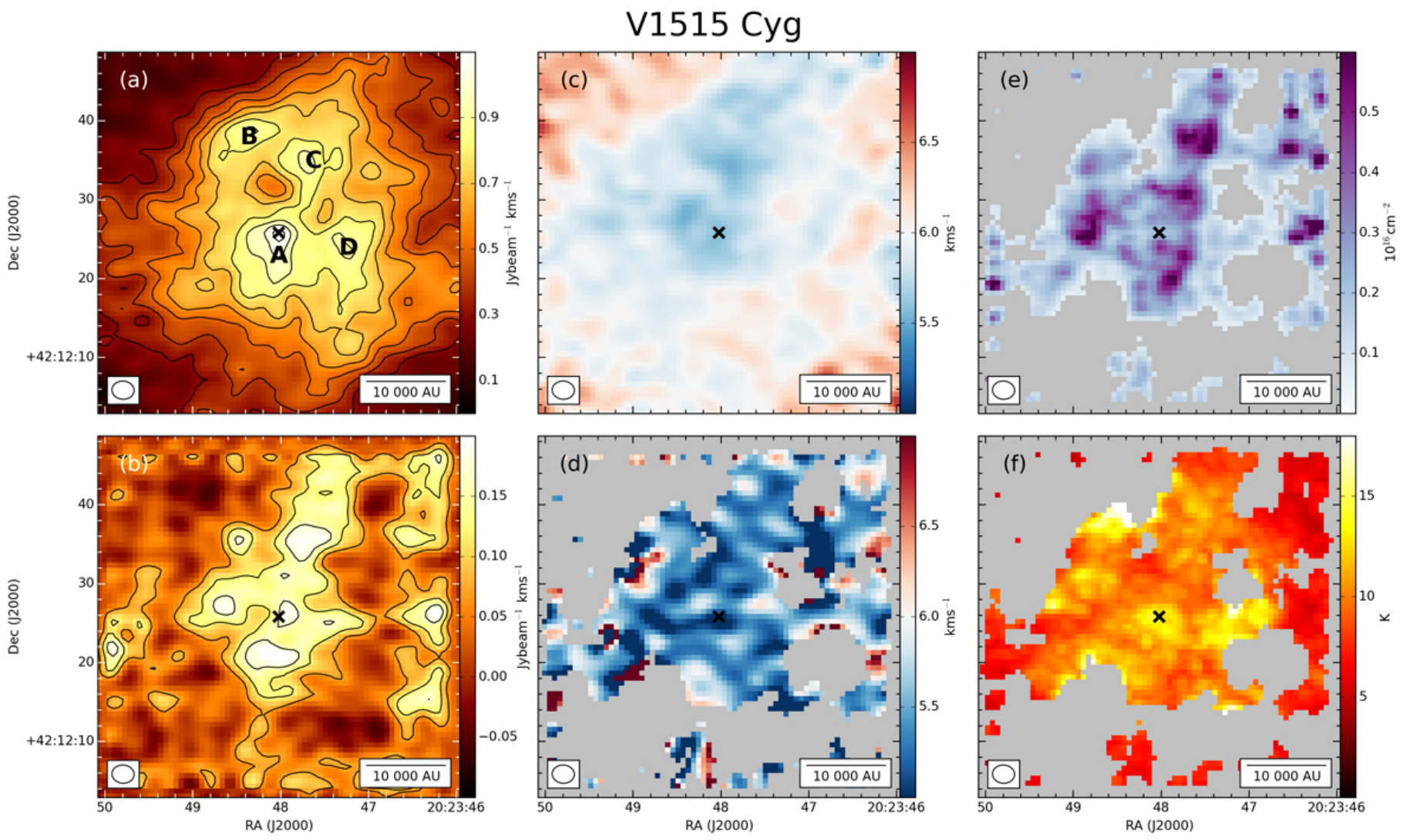

Fig. 5. Moment and physical parameter maps of V1515 Cyg. The panels are the same as in Fig. 3, the position of the star and the clumps marked the same. $\sigma_{\text {int }}=0.1 \mathrm{Jy} \mathrm{beam}^{-1} \mathrm{~km} \mathrm{~s}^{-1}$ and $n=3,4, \ldots, 12$ on $(a)$ and $\sigma_{\text {int }}=0.024 \mathrm{Jy} \mathrm{beam}^{-1} \mathrm{~km} \mathrm{~s}^{-1}$ and $n=3,5,7$ on $(b)$. The pixels with $\mathrm{C}^{18} \mathrm{O}$ peak values less than $9 \sigma$ are coloured grey on panels $d, e$ and $f$.

the centre with $1.7 \mathrm{Jybeam}^{-1} \mathrm{~km} \mathrm{~s}^{-1}$ in clump A. The similarly bright clump B is found at $7.3^{\prime \prime}$ to the northwest and the smaller clump $\mathrm{C}$ is inside a protrusion at $10^{\prime \prime}$ to the west. Two other small clumps are located close to the western edge of the map (E and F). The more diffuse emission reaches beyond the map edge towards the south, containing at least two other clumps (D and G). The $\mathrm{C}^{18} \mathrm{O}$ line integrated intensity distribution shows the same features but with only two significant clumps in the centre region, that coincide with clumps A and $\mathrm{B}$ with peaks of 0.46 and $0.48 \mathrm{Jy} \mathrm{beam}^{-1} \mathrm{~km} \mathrm{~s}^{-1}$. The smaller clumps at the western and southern edges of the map also appear.

The overall distribution of the emission seems to be moving from the southwest to the northeast on the channel maps of both isotopologues, and this appears as a large-scale velocity gradient of $0.05 \mathrm{~km} \mathrm{~s}^{-1} \operatorname{arcsec}^{-1}\left(18.7 \mathrm{~km} \mathrm{~s}^{-1} \mathrm{pc}^{-1}\right.$ at $\left.550 \mathrm{pc}\right)$ across the position of the star from the west to the east (Figs. 6c and d). At low velocities there is emission only to the southern-southwestern edge of the map in the ${ }^{13} \mathrm{CO}$ channel maps (Fig. A.5), but above $4.18 \mathrm{~km} \mathrm{~s}^{-1}$ four clumps form in a linear, northwest-southeast configuration. The two northern and two southern clumps merge, forming clumps $\mathrm{A}-\mathrm{D}$ and at around these same channels clump E, F and G emerge as well. The clumps A-D stay in the same formation between 4.82 and $5.03 \mathrm{~km} \mathrm{~s}^{-1}$ and they start to merge into one structure above $5 \mathrm{~km} \mathrm{~s}^{-1}$, that is centred somewhat to the east from the centre. Above $5.67 \mathrm{~km} \mathrm{~s}^{-1}$ only two bright clumps are present, clumps A and $\mathrm{B}$.

The eastern edge of the elongated ${ }^{13} \mathrm{CO}$ structure coincides with the bright rim of $\mathrm{H} \alpha$ nebulosity observed by PTF
(Hillenbrand et al. 2013). The CO emission starts just inside the nebulosity and also coincides with the bright region around the FUor in Herschel images (Kóspál et al. 2013). This structure may indicate a shock front edge, formed by the same ionising radiation that causes the bright rim in $\mathrm{H} \alpha$, compressing the material around V2492 Cyg.

The densest region on the $N_{\mathrm{C}^{18} \mathrm{O}}$ map is directly behind the shock front in an arc-like structure, including clump A and B with peaks of 1.6 and $2.6 \times 10^{16} \mathrm{~cm}^{-2}$. Clump A also seems to be heated to $30 \mathrm{~K}$ in contrast with the $20 \mathrm{~K}$ temperatures on most of the mapped area. Two other warmer spots appear on the temperature maps with 55 and $70 \mathrm{~K}$, one roughly coincides with clump C and the other is in the middle of the triangle of clumps A, B and C. Clump A coincides with the strong continuum source that appears at $2.7 \mathrm{~mm}$. The "tail"-shaped continuum feature points towards clump $\mathrm{C}$ but does not extend that far. The other, faint continuum point source is located to the south from clump C. A third $2.7 \mathrm{~mm}$ source was detected by Hillenbrand et al. (2013) which does not appear in our continuum map but it would roughly coincide with our clump D on the CO maps. Clump B does not appear in the continuum, despite it being the hottest and densest source in our CO maps. Using $23.5 \mathrm{~K}$ and $29.8 \mathrm{~K}$ average clump temperatures and radii of $3.5^{\prime \prime}$ we calculate the masses of clump $\mathrm{A}$ and $\mathrm{B}$ as 0.17 and $0.23 M_{\odot}$, while clump $\mathrm{C}$ has a mass of $0.09 M_{\odot}$, using $26.7 \mathrm{~K}$ and $3^{\prime \prime}$.

\subsection{V2493 Cyg}

Both ${ }^{13} \mathrm{CO}$ and $\mathrm{C}^{18} \mathrm{O}$ show wide, multi-peaked lines and some excess mainly on the blue line wings. The ${ }^{13} \mathrm{CO}$ integrated 

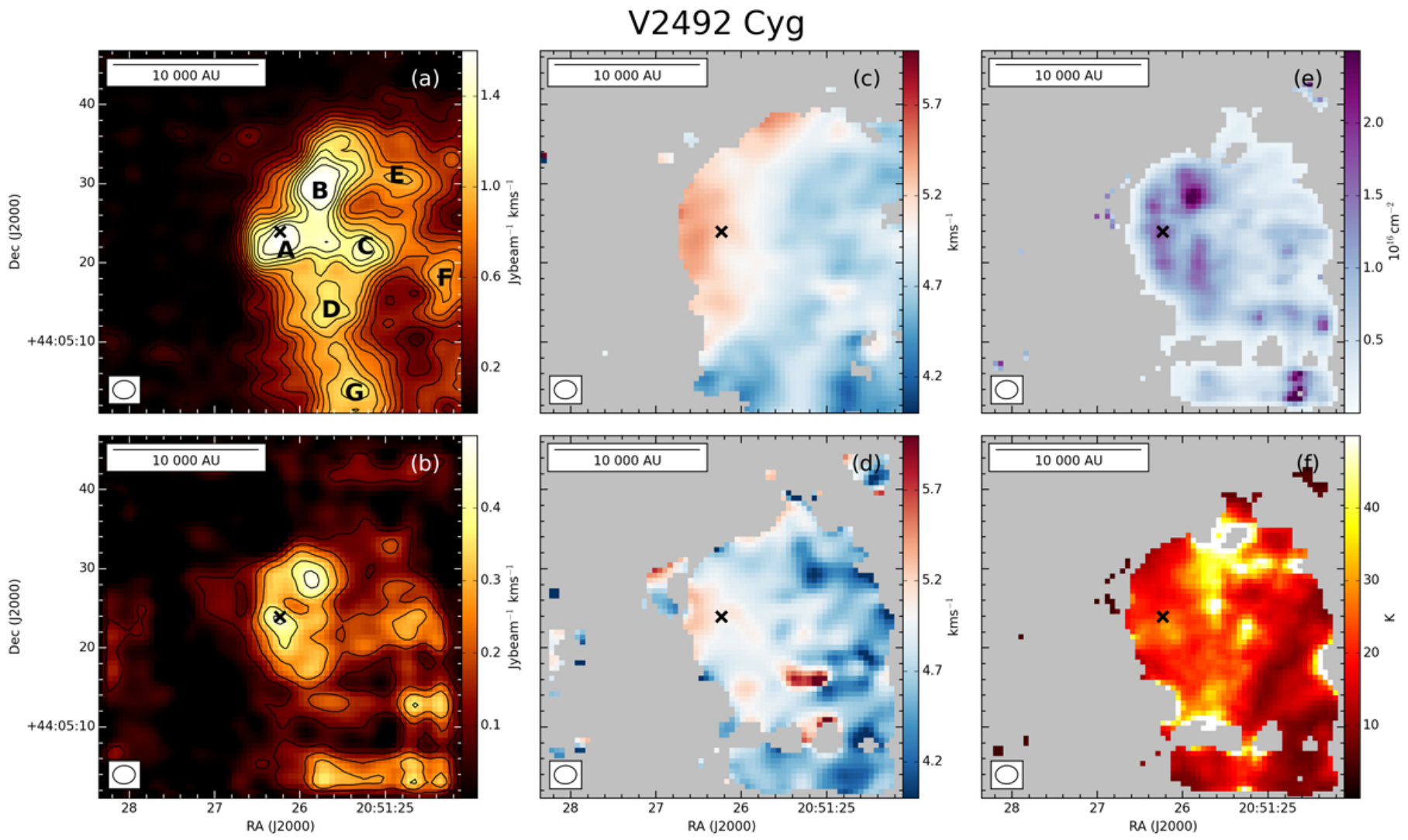

Fig. 6. Moment and physical parameter maps of V2492 Cyg. The panels are the same as in Fig. 3, the position of the star and the clumps marked the same. $\sigma_{\text {int }}=0.04 \mathrm{Jy} \mathrm{beam}^{-1} \mathrm{~km} \mathrm{~s}^{-1}$ and $n=3,6, \ldots, 36$ on $(a)$ and $\sigma_{\text {int }}=0.03 \mathrm{Jy}_{\text {beam }}{ }^{-1} \mathrm{~km} \mathrm{~s}^{-1}$ and $n=3,6, \ldots, 18$ on $(b)$. The pixels with $\mathrm{C}^{18} \mathrm{O}$ peak values less than $9 \sigma$ are coloured grey on panels $d, e$ and $f$.

intensity map shows an extended, roughly circular, $20^{\prime \prime} \times 30^{\prime \prime}$ bright area around the star with several little clumps (Fig. 7a). The elongated clump A has two peaks north from the centre with $2.6 \mathrm{Jy}_{\text {beam }}^{-1} \mathrm{~km} \mathrm{~s}^{-1}$. There are three brighter (B-D) and two fainter clumps (E and $\mathrm{F})$ around. These clumps appear above $2.6 \mathrm{~km} \mathrm{~s}^{-1}$ in the channel maps (Fig. A.7), then fragment and merge throughout the whole velocity interval. Clump A is in fact two small clumps close to each other, the northern at lower velocities than the southern and this appears as a velocity gradient of $0.06 \mathrm{~km} \mathrm{~s}^{-1} \operatorname{arcsec}^{-1}\left(22.5 \mathrm{~km} \mathrm{~s}^{-1} \mathrm{pc}^{-1}\right.$ at $\left.550 \mathrm{pc}\right)$ in the clump. Clump E corresponds to the $2.7 \mathrm{~mm}$ continuum source MMS1 (Dunham et al. 2012). We also detected MMS2-MMS4 in our continuum maps but none of them correspond to any of the CO clumps. The FUor itself does not appear in the continuum and even in ${ }^{13} \mathrm{CO}$, emission centred on it can only be observed by integrating between 5.45 and $6.52 \mathrm{~km} \mathrm{~s}^{-1}$, as shown by Kóspál et al. (2016). The $\mathrm{C}^{18} \mathrm{O}$ integrated intensity map (Fig. 7c) shows only weak emission, the peak is at $4^{\prime \prime}$ to the southwest from the centre with $0.3 \mathrm{Jy}_{\text {beam }}{ }^{-1} \mathrm{~km} \mathrm{~s}^{-1}$. The $\mathrm{C}^{18} \mathrm{O}$ channel maps (Fig. A.8) are similar to the overall distribution of the ${ }^{13} \mathrm{CO}$ emission. It is difficult to recognise clumps here, except between 4.82 and $5.03 \mathrm{~km} \mathrm{~s}^{-1}$ where a point-like, bright source appears at $6^{\prime \prime}$ to the northwest from the centre. This source is close to clump A but does not coincide with it.

As seen in Fig. 2 the three peaks on the ${ }^{13} \mathrm{CO}$ line profile are around the same velocities as the peaks on the $\mathrm{C}^{18} \mathrm{O}$ line, thus they may be in fact three line components centred on $3.4,5.1$ and $7.1 \mathrm{~km} \mathrm{~s}^{-1}$. Integrating the emission in the ranges $0.3-4.3$, 4.3-6.5 and $6.5-8.7 \mathrm{~km} \mathrm{~s}^{-1}$ it is apparent that the lower velocity line traces mainly clumps A, B and D, the middle ones clumps A, C and $\mathrm{E}$ and the high velocity line component shows weaker, clumpy emission throughout the whole area.

The derived temperatures are generally $10-20 \mathrm{~K}$, with higher peaks that are mainly due to the lower $\mathrm{S} / \mathrm{N}$ of the $\mathrm{C}^{18} \mathrm{O}$ spectra. The $\mathrm{C}^{18} \mathrm{O}$ column densities are generally around $1-6 \times 10^{15} \mathrm{~cm}^{-2}$ and rise to $2.7 \times 10^{16} \mathrm{~cm}^{-2}$ at the point-sourcelike object to the northwest. Here even $\tau_{18}$ is close to unity. The mass of the clump around the FUor integrated between 5.45 and $6.52 \mathrm{~km} \mathrm{~s}^{-1}$ with a radius of $3^{\prime \prime}$ is $0.024 M_{\odot}$. Since temperatures are not well measured around the centre, we use here an average $T=21.6 \mathrm{~K}$ calculated inside the primary beam. The clumps A-D have masses of $0.05-0.15 M_{\odot}$ using the same temperature.

\subsection{V1735 Cyg}

Both the ${ }^{13} \mathrm{CO}$ and $\mathrm{C}^{18} \mathrm{O}$ lines in V1735 Cyg show at least two peaks and excess emission on both line wings. In the ${ }^{13} \mathrm{CO}$ integrated intensity map an elliptic, bright area appears that is elongated in the northeast-southwest direction (Fig. 8a). The southeastern part of the structure is fainter than the northwestern. The emission peak (2.3 Jy beam ${ }^{-1} \mathrm{~km} \mathrm{~s}^{-1}$ ) coincides with the FUor inside a north-south elongated, bright, elliptic clump that we call clump A. There is a weaker clump to the northeast that protrudes from this central area (clump B). The point source detected in the $2.7 \mathrm{~mm}$ continuum roughly coincides with clump A, but the weaker continuum features around the strong detection have no counterparts in the $\mathrm{CO}$ maps. In the ${ }^{13} \mathrm{CO}$ channel maps (Fig. A.9) an S-shaped region of emission appears at the centre between 2.41 and $3.15 \mathrm{~km} \mathrm{~s}^{-1}$ that breaks up into several 

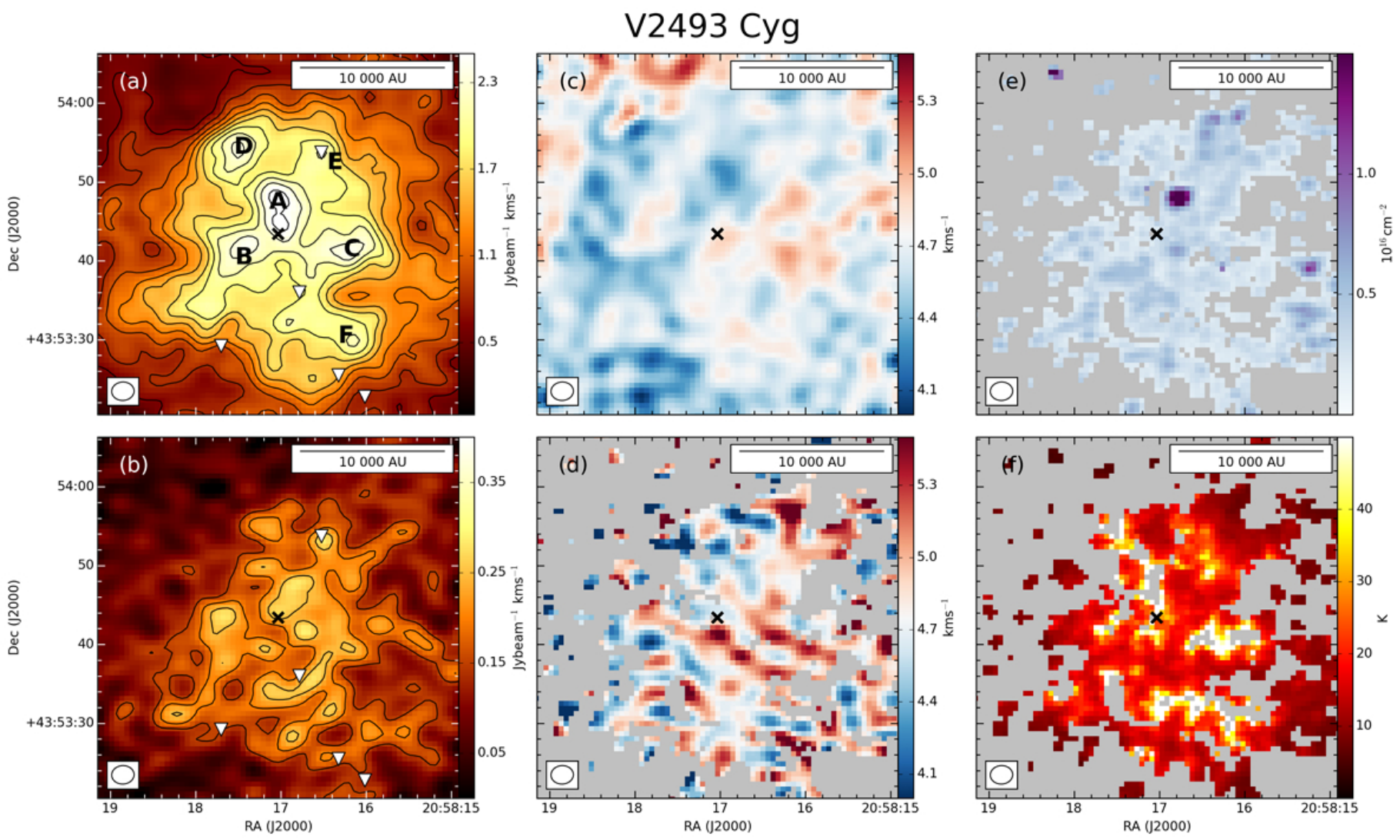

Fig. 7. Moment and physical parameter maps of V2493 Cyg. The panels are the same as in Fig. 3, the position of the star and the clumps marked the same. $\sigma_{\text {int }}=0.21 \mathrm{Jy}_{\text {beam }}^{-1} \mathrm{~km} \mathrm{~s}^{-1}$ and $n=3,4, \ldots, 12$ on $(a)$ and $\sigma_{\text {int }}=0.05 \mathrm{Jy} \mathrm{beam}^{-1} \mathrm{~km} \mathrm{~s}^{-1}$ and $n=3,4, \ldots, 8$ on $(b)$. The triangles mark the millimetre sources from Dunham et al. (2012) as in Fig. 1. The pixels with $\mathrm{C}^{18} \mathrm{O}$ peak values less than $7 \sigma$ are coloured grey on panels $d, e$ and $f$.

clumps. Between 3.68 and $4.32 \mathrm{~km} \mathrm{~s}^{-1}$ there are three bright regions at the centre that form another bright S-shaped structure which then breaks up again: the southwestern and northeastern parts disappear by $4.64 \mathrm{~km} \mathrm{~s}^{-1}$ and only the central clump is significant at higher velocities. Weaker emission is present to the north even above $8 \mathrm{~km} \mathrm{~s}^{-1}$. The $\mathrm{C}^{18} \mathrm{O}$ integrated intensity map (Fig. 8c) is very different from ${ }^{13} \mathrm{CO}$, showing significant emission only on the north side of the star with a peak of $0.4 \mathrm{Jy}_{\text {beam }}{ }^{-1} \mathrm{~km} \mathrm{~s}^{-1}$. The reason for this can be seen on the channel maps (Fig. A.10), where the same S-shaped emission appears but the north-northwestern side of the structure is much brighter. Above $3.79 \mathrm{~km} \mathrm{~s}^{-1}$ only an irregularly shaped central source is present, somewhat to the northwest from the centre.

The velocity map of ${ }^{13} \mathrm{CO}$ (Fig. 8c) shows somewhat higher values in clump A than in the rest of the elliptical structure and there is a high-velocity lobe to the north. This is caused by emission appearing at $8-9 \mathrm{~km} \mathrm{~s}^{-1}$ velocities in the channel maps. The linewidth distribution also shows two areas with broad lines $\left(4.5-5.5 \mathrm{~km} \mathrm{~s}^{-1}\right)$ to the northwest and to the southeast of the star. The northern one coincides with the northern end of the highvelocity lobe. Integrating the ${ }^{13} \mathrm{CO}$ line wings between -0.2 and $2 \mathrm{~km} \mathrm{~s}^{-1}$ and between 7 and $9.4 \mathrm{~km} \mathrm{~s}^{-1}$, it is apparent that the blue and red-shifted areas occupy two distinct regions to the south and north of the centre, respectively (Fig. 11). The high linewidth regions are spatially connected to the lobes, suggesting the presence of an outflow. The orientation of the lobes agrees with the outflow morphology detected by Evans et al. (1994) in ${ }^{12} \mathrm{CO}$, although their angular resolution was much lower and they mapped a larger area. The outflow was proposed to be driven by the other continuum source at $20^{\prime \prime}$ to the northeast but the geometry of the lobes detected here suggests that it may be driven by V1735 Cyg.

Around the region with high ${ }^{13} \mathrm{CO}$ linewidths, the $\mathrm{C}^{18} \mathrm{O}$ emission is very bright, while ${ }^{13} \mathrm{CO}$ is very strongly selfabsorbed, causing their line ratio to be higher than in the optically thick limit. This results in very high or undefinable optical depths in the area. The $\mathrm{C}^{18} \mathrm{O}$ column densities are significantly higher here than to the south: it is generally around $0.5-2.3 \times 10^{16} \mathrm{~cm}^{-2}$ with no clear peak at the centre. The ${ }^{13} \mathrm{CO}$ optical depth also shows somewhat higher values to the north (4-10) than to the south (1-4). The temperatures are around $15-20 \mathrm{~K}$ in the elliptical structure and peak to the south from the centre with 30 and $33 \mathrm{~K}$. We measure $25 \mathrm{~K}$ at the FUor position. We calculated the dust optical depth from the continuum map by subtracting a Planck-function defined by the derived gas temperatures pixel-by-pixel and this shows that the strong continuum source is mainly a temperature effect and there is not a high-density structure in the map, similarly to the $\mathrm{C}^{18} \mathrm{O}$ column density distribution. The gas-based mass of clump A with a radius of $4^{\prime \prime}$ and an average temperature of $19.7 \mathrm{~K}$ is $0.51 M_{\odot}$.

\subsection{V733 Cep}

Our observations of V733 Cep show self-absorbed ${ }^{13} \mathrm{CO}$ emission with some excess on the blue line wing. The ${ }^{13} \mathrm{CO}$ integrated intensity peak is located at $4^{\prime \prime}$ to the northeast from the FUor, with $1.5 \mathrm{Jy} \mathrm{beam}^{-1} \mathrm{~km} \mathrm{~s}^{-1}$, in clump A (Fig. 9a). There is another, fainter clump to the southwest, clump B. In the ${ }^{13} \mathrm{CO}$ channel maps at low velocities there is only significant emission in the southwestern corner, then an elongated structure 

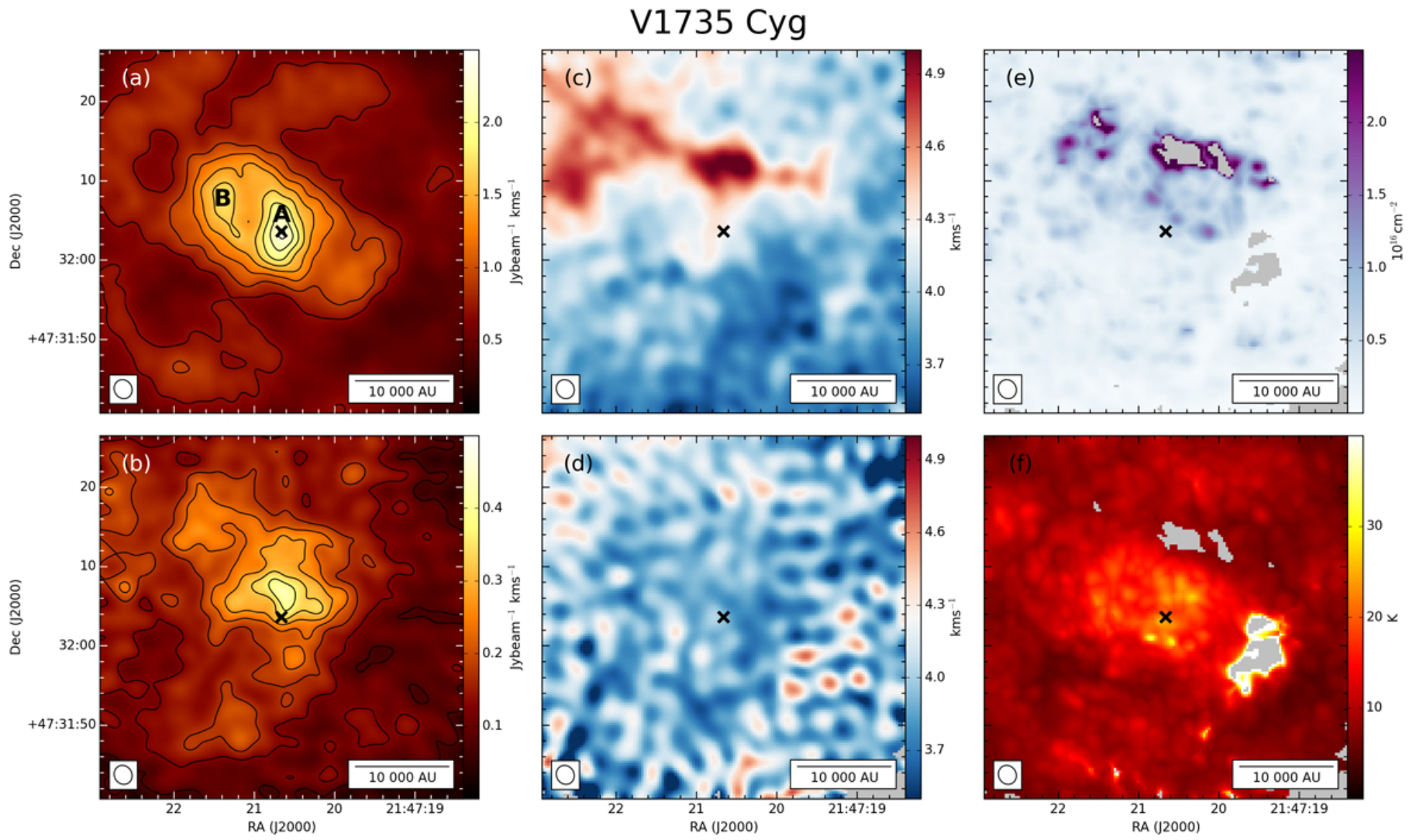

Fig. 8. Moment and physical parameter maps of V1735 Cyg. The panels are the same as in Fig. 3, the position of the star and the clumps marked the same. $\sigma_{\text {int }}=0.23 \mathrm{Jy} \mathrm{beam}^{-1} \mathrm{~km} \mathrm{~s}^{-1}$ and $n=3,4, \ldots, 13$ on $(a)$ and $\sigma_{\text {int }}=0.025 \mathrm{Jy} \mathrm{beam}^{-1} \mathrm{~km} \mathrm{~s}^{-1}$ and $n=3,5, \ldots, 15$ on $(b)$. The pixels with $\mathrm{C}^{18} \mathrm{O}$ peak values less than $18 \sigma$ are coloured grey on panels $d, e$ and $f$.

appears in the northeast-southwest direction, between -10.2 and $-9.77 \mathrm{~km} \mathrm{~s}^{-1}$. Clump A appears around $-9.77 \mathrm{~km} \mathrm{~s}^{-1}$ and it is significant until $-8.08 \mathrm{~km} \mathrm{~s}^{-1}$, somewhat changing its position and size. Clump B is present in these channels as well. Other small clumps appear further northeast, to the south and to the southwest. At $-8.71 \mathrm{~km} \mathrm{~s}^{-1}$ there are only three strong clumps and above $-7.86 \mathrm{~km} \mathrm{~s}^{-1}$ there is no significant emission in the centre. However, even at $-6 \mathrm{~km} \mathrm{~s}^{-1}$ there is emission in the southeastern region of the map. There is no clear systematic change in the line velocities but ${ }^{13} \mathrm{CO}$ bright areas generally have higher velocities and smaller line widths $\left(2-2.5 \mathrm{~km} \mathrm{~s}^{-1}\right)$ than the background.

The $\mathrm{C}^{18} \mathrm{O}$ line integrated intensity map shows emission mainly to the north of the source, since between $-9.35 \mathrm{~km} \mathrm{~s}^{-1}$ and -8.5 the strongest $\mathrm{C}^{18} \mathrm{O}$ emission comes from the brightest ${ }^{13} \mathrm{CO}$ clumps, to the northeast, north and northwest. Similarly to V1515 Cyg the ${ }^{13} \mathrm{CO}$ channel maps might be interpreted as a small expanding shell with the receding side seen around $-9.77 \mathrm{~km} \mathrm{~s}^{-1}$ and the approaching wall around $-8.08 \mathrm{~km} \mathrm{~s}^{-1}$. This is supported by the fact that there is no $\mathrm{C}^{18} \mathrm{O}$ emission peak towards the FUor in any of the channels.

The temperatures are generally higher inside the bright ${ }^{13} \mathrm{CO}$ emission areas than at the edges of the map, with $10-20 \mathrm{~K}$ and peaks of 45 and $75 \mathrm{~K}$ to the northeast. The high opacity filaments to the north seem to be cold, around $10 \mathrm{~K}$. We measure higher $N_{\mathrm{C}^{18} \mathrm{O}}$ values to the north than the south, with peaks of 4.8 and $7 \times 10^{15} \mathrm{~cm}^{-2}$. Clump A with an average $T=27.3 \mathrm{~K}$ has a mass of $0.22 M_{\odot}$ and clump B with $T=17.9 \mathrm{~K}$ has a mass of $0.06 M_{\odot}$.

Integrating the ${ }^{13} \mathrm{CO}$ emission in the line wings between -12.9 and $-10.7 \mathrm{~km} \mathrm{~s}^{-1}$, then between -7.4 and $-6 \mathrm{~km} \mathrm{~s}^{-1}$ (see
Fig. 11), the blue wing shows emission mostly to the southwest and the red wing to the southeast, in a somewhat clumpy structure. If this indeed indicates an outflow, the source that drives it should be located around $20^{\prime \prime}$ to the south of the centre, rather than V733 Cep itself.

\subsection{V710 Cas}

Both the ${ }^{13} \mathrm{CO}$ and $\mathrm{C}^{18} \mathrm{O}$ lines towards V710 Cas (RNO 1B) show multiple peaks and possibly self-absorption. The ${ }^{13} \mathrm{CO}$ integrated intensity map has a bright peak at $2.5^{\prime \prime}$ to the northeast of RNO $1 \mathrm{~B}$ with $4.6 \mathrm{Jy} \mathrm{beam}^{-1} \mathrm{~km} \mathrm{~s}^{-1}$ (Fig. 10a). This circular bright area that we call clump A is located between RNO 1B and $\mathrm{C}$. Further peaks can be found at the end of an arc-like structure to the west (clump B), little local peaks to the east and the much fainter clump $\mathrm{C}$ to the south. The $\mathrm{C}^{18} \mathrm{O}$ integrated intensities show the same morphology with a peak of $1 \mathrm{Jy} \mathrm{beam}^{-1} \mathrm{~km} \mathrm{~s}^{-1}$ at $3.8^{\prime \prime}$ from RNO $1 \mathrm{~B}$ that roughly coincides with the ${ }^{13} \mathrm{CO}$ peak. Both the ${ }^{13} \mathrm{CO}$ and the $\mathrm{C}^{18} \mathrm{O}$ velocity maps show clump $\mathrm{A}$ and the eastern side of the arc at higher velocities than clump B, with a gradient of $0.1 \mathrm{~km} \mathrm{~s}^{-1} \operatorname{arcsec}^{-1}$ from the east to the west (corresponding to $25.7 \mathrm{~km} \mathrm{~s}^{-1} \mathrm{pc}^{-1}$ at $800 \mathrm{pc}$ ). Indeed at low velocities the ${ }^{13} \mathrm{CO}$ channel maps show a westsouth elongated clump where first clump B then clump A becomes more brighter. Between -18.41 and $-17.87 \mathrm{~km} \mathrm{~s}^{-1}$ several clumps form an $\mathrm{S}$-shaped structure and above $-17.78 \mathrm{~km} \mathrm{~s}^{-1}$ the western arc of the structure starts to disintegrate. Around $-17.35 \mathrm{~km} \mathrm{~s}^{-1}$ only clump $\mathrm{C}$ and extended emission roughly around clump A are present. At high velocities clump A becomes 

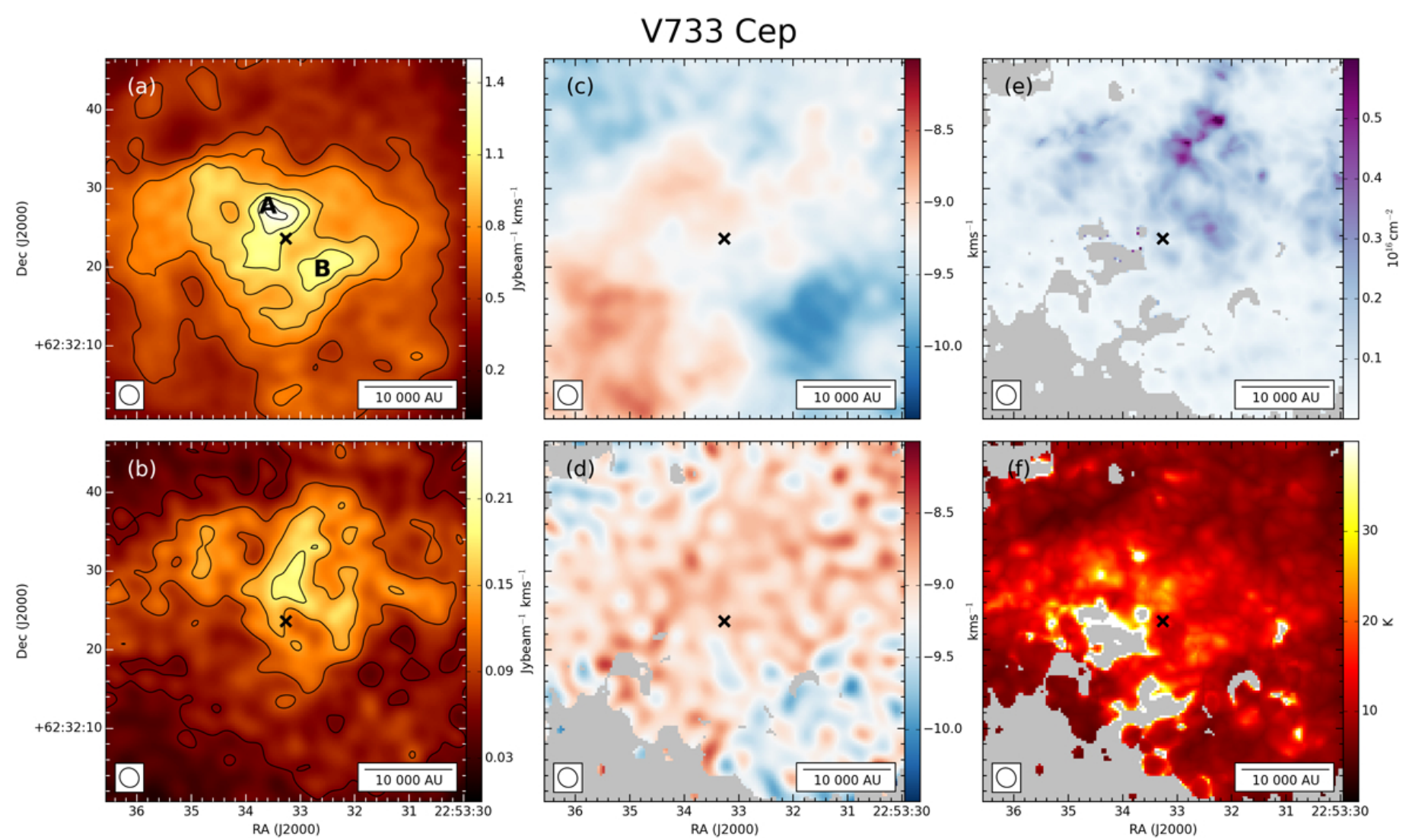

Fig. 9. Moment and physical parameter maps of V733 Cep. The panels are the same as in Fig. 3, the position of the star and the clumps marked the same. $\sigma_{\text {int }}=0.18 \mathrm{Jy} \mathrm{beam}^{-1} \mathrm{~km} \mathrm{~s}^{-1}$ and $n=3,4, \ldots, 8$ on $(a)$ and $\sigma_{\text {int }}=0.02 \mathrm{Jy}_{\text {beam }}{ }^{-1} \mathrm{~km} \mathrm{~s}^{-1}$ and $n=3,5, \ldots, 9$ on $(b)$. The pixels with $\mathrm{C}^{18} \mathrm{O}$ peak values less than $10 \sigma$ are coloured grey on panels $d, e$ and $f$.

brighter again and another clump appears at $10^{\prime \prime}$ to the northeast at $-16.29 \mathrm{~km} \mathrm{~s}^{-1}$. The two clumps are present until around $-14.9 \mathrm{~km} \mathrm{~s}^{-1}$.

Emission at the centre is present between -19.6 and $-16.7 \mathrm{~km} \mathrm{~s}^{-1}$ but it is never centred on RNO 1B. Clump A coincides with one of the sources detected in the $2.7 \mathrm{~mm}$ continuum. The shape of this source suggests that it may be the superposition of smaller objects and one of those might correspond to RNO 1C. This continuum source roughly coincides with RNO 1D measured by Weintraub \& Kastner (1993) and with VLA 1 detected by Anglada et al. (1994) as well. The brightest continuum source to the northeast coincides with IRAS $00338+6312$ and with a small ${ }^{13} \mathrm{CO}$ and $\mathrm{C}^{18} \mathrm{O}$ clump. This source was also detected as VLA 3 by Anglada et al. (1994). VLA 2 was also detected in the continuum and in ${ }^{13} \mathrm{CO}$ as well to the southeast from RNO 1B. It also coincides with RNO 1 G Weintraub \& Kastner (1993). The brighter source to the west of it has no counterpart in the $\mathrm{CO}$ maps and was not measured in IR or sub-millimetre before. We did not detect RNO $1 \mathrm{~F}$.

Integrating the ${ }^{13} \mathrm{CO}$ line wing emission between -23.3 and $-19.6 \mathrm{~km} \mathrm{~s}^{-1}$, then between -15.6 and $-13.3 \mathrm{~km} \mathrm{~s}^{-1}$ (Fig. 11) the blue lobe extends mainly to the west-southwest and the red lobe consists of two distinct clumps to the east-northeast from the centre. The orientation of the lobes mark the same axis as the outflow detected by Evans et al. (1994) in ${ }^{12} \mathrm{CO}$ and by Yang et al. (1995) in CS. The same structure can be seen by integrating the $\mathrm{C}^{18} \mathrm{O}$ line wings. However, the emission at these low and high velocities could be simply originating in clumps instead of an outflow.

The temperature distribution in the area does not show a clear peak around the centre but we see values mostly above $40-50 \mathrm{~K}$ along the arc-shaped structure in the ${ }^{13} \mathrm{CO}$ map and values of $20-30 \mathrm{~K}$ to the south and on both sides of RNO $1 \mathrm{~B}$. The $\mathrm{C}^{18} \mathrm{O}$ column density map shows higher peaks mainly around and between clump A, IRAS 00338+6312 and clump B with values above $5 \times 10^{16} \mathrm{~cm}^{-2}$. Similarly to V1735 Cyg, calculating the millimetre optical depths shows that except IRAS $00338+6312$ all the other continuum sources are bright due to a temperature effect and not a significant density enhancement. Using the $\mathrm{C}^{18} \mathrm{O}$ emission, clump A with $T=38 \mathrm{~K}$ average temperature has a mass of $1.8 M_{\odot}$ and clump B with $T=41 \mathrm{~K}$ has a mass of $0.77 M_{\odot}$.

\section{Discussion}

The CO emission around all our targets shows an extended structure of several thousand $\mathrm{AU}$ in diameter, where the intensity of the emission decreases more steeply or less steeply towards the edge of the primary beam area. Inside this area the distribution of the CO emission is mostly clumpy and complex. There are two cases where the detected ${ }^{13} \mathrm{CO}$ emission has one significant peak that is centred on the targeted FUors: around V1057 Cyg with a radius of $3000 \mathrm{AU}$ and a mass of $0.21 M_{\odot}$ and around V1735 Cyg with a radius of $3400 \mathrm{AU}$ and a mass of $0.51 M_{\odot}$. These clumps are roughly spherical, clearly heated by the central stars, show strong density enhancements and are also detected in the continuum. Similar clumps were detected centred on V1515 Cyg, V2492 Cyg and V2493 Cyg with masses of $0.26,0.17$ and $0.024 M_{\odot}$, respectively, but the distribution of the $\mathrm{CO}$ emission around these FUors has multiple peaks and shows several clumps. The central clumps of V1515 Cyg and 

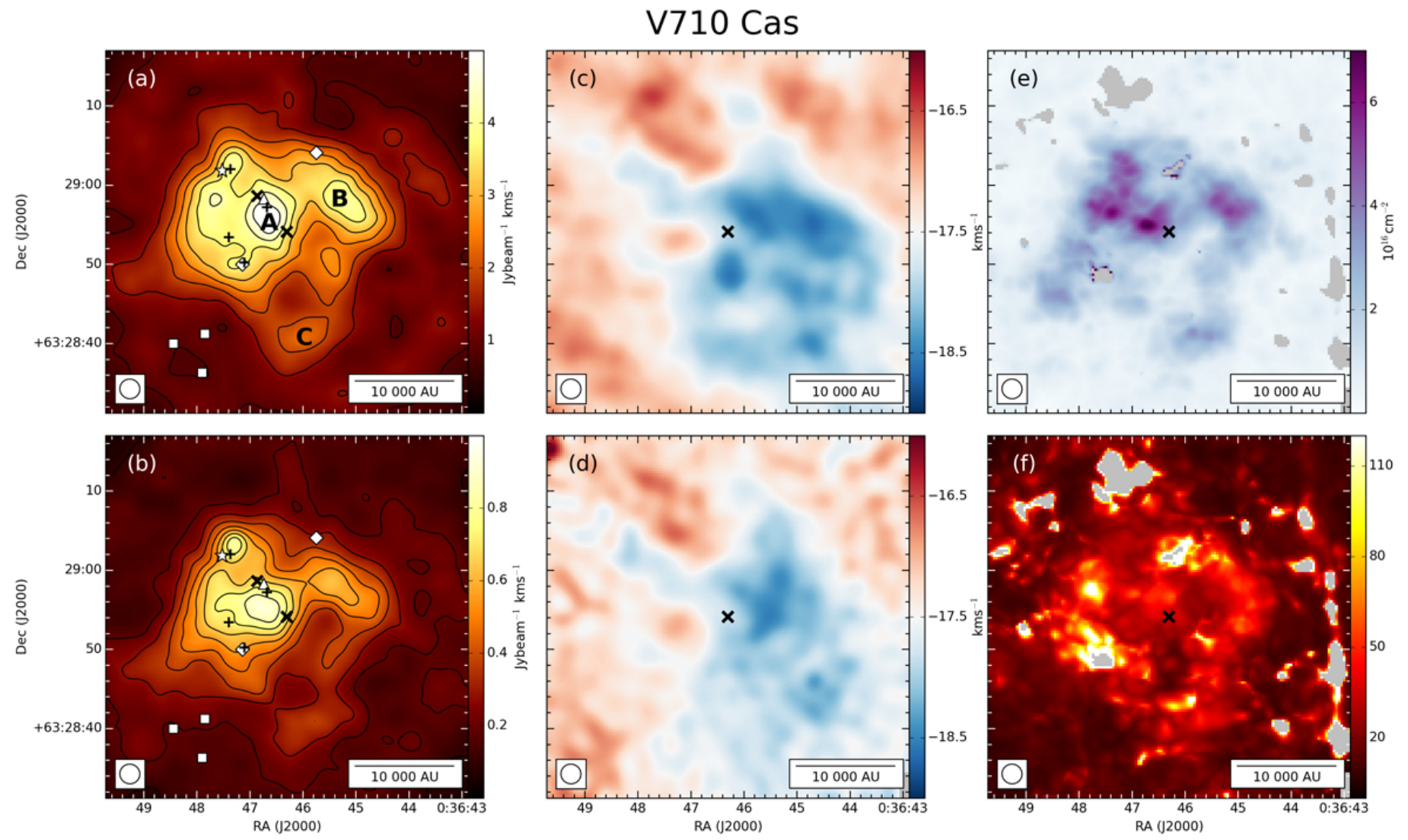

Fig. 10. Moment and physical parameter maps of V710 Cas. The panels are the same as in Fig. 3, the position of the star and the clumps marked the same. $\sigma_{\text {int }}=0.28 \mathrm{Jy} \mathrm{beam}^{-1} \mathrm{~km} \mathrm{~s}^{-1}$ and $n=3,5, \ldots, 17$ on $(a)$ and $\sigma_{\text {int }}=0.031 \mathrm{Jy}_{\text {beam }}{ }^{-1} \mathrm{~km} \mathrm{~s}^{-1}$ and $n=3,6, \ldots, 33$ on $(b)$. The previously detected sources are marked similarly as in Fig. 1. The pixels with $\mathrm{C}^{18} \mathrm{O}$ peak values less than $18 \sigma$ are coloured grey on panels $d, e$ and $f$.

V2492 Cyg are heated and these two are detected in the continuum as well. No CO clumps centred on V733 Cep and V710 Cas were found but other structures do appear in these areas. In the case of V710 Cas, clump A is located between the two members of the FUor-system, RNO 1B and RNO 1C. This clump is detected also in the continuum. Several other CO clumps in the area also coincide with sub-millimetre, IR or radio objects in previous measurements.

Considering the match between the detected CO line velocities and the characteristic velocities of the respective star forming regions, the extended circumstellar clouds that roughly fill the primary beam $\left(45.8^{\prime \prime}\right.$ diameter that corresponds to radii of 12600-22900 AU, depending on the distance) around the targeted FUors are clearly associated with the stars (Dobashi et al. 1994; Yonekura et al. 1997). Calculating the $M_{\text {total }}$ total mass inside the primary beam using an average temperature we get masses in the range $1-20 M_{\odot}$ (Table 4 ). Clearly most of the material on this spatial scale will not end up accreting onto the central star, thus these circumstellar structures are not identified as real envelopes. For V1057 Cyg, V1515 Cyg, V2492 Cyg and V1735 Cyg we find smaller scale clumps coinciding with the position of the star that are heated by the FUors; here we define these as envelopes. In the case of V2493 Cyg the temperatures are not well constrained and we only detect a small envelope on certain velocities that coincide with the star. In the case of V733 Cep and V710 Cas there is no small-scale clump coinciding with the FUors. The presence of many other objects around, for example, V710 Cas and V2493 Cyg, inside the detected extended circumstellar clouds also reinforces the idea that these are associated clouds but not real envelopes. We also note that the cleaning process of the maps may cause artifacts and noisy areas at the edge of the primary beam in the maps. For the targets where the ${ }^{13} \mathrm{CO}$ and $\mathrm{C}^{18} \mathrm{O}$ emission is not as centrally peaked (e.g. at V1515 Cyg, V2493 Cyg or V733 Cep) as at V1057 Cyg or V1735 Cyg, this might cause some confusion in determining the radius of the extended structure. It could happen that we see only a part of a much larger cloud that is cut off by the primary beam and the cleaning process into a primary beam sized, circular structure. This however is less likely, since we do not see increased noise at the edges, but a clear drop in intensity in all directions. Thus the detected extended clouds are probably real. The central temperature, density, the average temperature and density and the masses of the detected envelopes defined above are summarised in Table 4.

According to previous ${ }^{12} \mathrm{CO}$ mapping and optical spectra, all our targets (or neighbouring sources) drive outflows (Evans et al. 1994; Levreault 1988). For three of them the density is enough that the lobes appear even on the ${ }^{13} \mathrm{CO}$ line wings in our observations. The outflow towards V1735 Cyg is clearly centred on the star, it is perpendicular to the major axis of the larger scale extended circumstellar cloud and generally oriented the same way as previous, lower resolution molecular studies showed. The line wings towards V710 Cas could just be blended line components or, considering the large number of IR and radio sources indentified in the region, showing outflow lobes centred on other stars. The situation might be similar towards V733 Cep where the extended outflow lobes are centred on an unknown source south from the FUor.

Both the continuum-based mass calculation and linebased mass and column density calculation depend strongly 

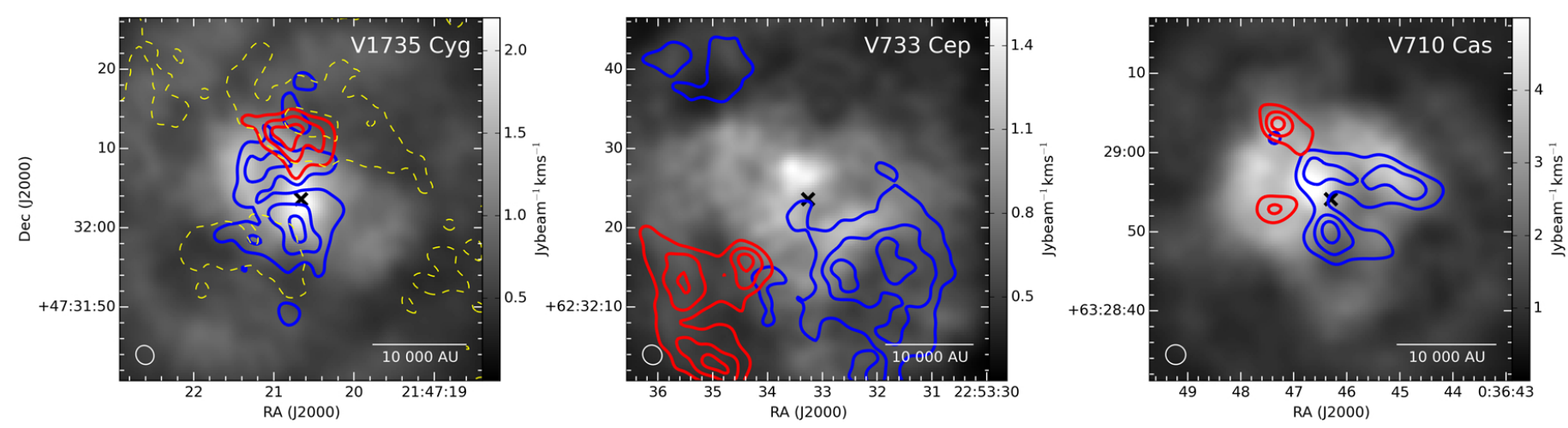

Fig. 11. Outflow features around V1735 Cyg, V733 Cep and V710 Cas. The grey-scale shows the integrated intensity of the line similarly as on the moment map, black crosses mark the position of the FUors and blue and red lines the integrated intensity contours on the line wings at 50,70 and $90 \%$ of the peak value. The line wings were integrated between the following velocities: the blue wing between -0.2 and $2 \mathrm{~km} \mathrm{~s}^{-1}$ and the red wing between 7 and $9.4 \mathrm{~km} \mathrm{~s}^{-1}$ towards V1735 Cyg; the blue wing between -12.9 and $-10.7 \mathrm{~km} \mathrm{~s}^{-1}$ and the red wing between -7.4 and $-6.0 \mathrm{~km} \mathrm{~s}{ }^{-1}$ towards V733 Cep; the blue wing between -23.3 and $-19.6 \mathrm{~km} \mathrm{~s}^{-1}$ and the red wing between -15.6 and $-13.3 \mathrm{~km} \mathrm{~s}^{-1}$ towards V710 Cas. The yellow dashed contours mark the ${ }^{13} \mathrm{CO}$ FWHM linewidth contours at 4 and $5 \mathrm{~km} \mathrm{~s}^{-1}$.

Table 4. Envelope masses and the silicate feature at out targets.

\begin{tabular}{clclccccc}
\hline \hline Target & $\begin{array}{c}T_{\mathrm{c}} \\
{[\mathrm{K}]}\end{array}$ & $\begin{array}{c}N_{\mathrm{c}} \\
{\left[10^{16} \mathrm{~cm}^{-2}\right]}\end{array}$ & $\begin{array}{l}T_{\text {env }}[\mathrm{K}] \\
{\left[10^{16} \mathrm{~cm}^{-2}\right]}\end{array}$ & $\begin{array}{c}R_{\text {env }} \\
{[\mathrm{AU}]}\end{array}$ & $\begin{array}{c}M_{\text {env }} \\
{\left[M_{\odot}\right]}\end{array}$ & $\begin{array}{c}M_{\text {total }} \\
{\left[M_{\odot}\right]}\end{array}$ & Si band \\
\hline V1057 Cyg & 17 & 0.81 & 16.4 & 0.84 & 3000 & 0.21 & 1.25 & em \\
V1515 Cyg & 14.2 & 0.25 & 11 & 0.29 & 5000 & 0.26 & 3.1 & em \\
V2492 Cyg & 24.1 & 1.68 & 23.5 & 1.10 & 1925 & 0.17 & 1.9 & $\ldots$ \\
V2493 Cyg & $\ldots$ & $\ldots$ & 37 & 0.29 & 1650 & 0.024 & 3.15 & $\ldots$ \\
V1735 Cyg & 24.5 & 0.41 & 19.7 & 0.51 & 3400 & 0.51 & 6.9 & abs \\
V733 Cep & 14 & 0.12 & $\ldots$ & $\ldots$ & $\ldots$ & $\ldots$ & 4.5 & abs \\
V710 Cas & 31.9 & 3.62 & $\ldots$ & $\ldots$ & $\ldots$ & $\ldots$ & 19.6 & abs \\
\hline
\end{tabular}

Notes. The columns are: (1) target name; (2) temperature at the target position; (3) $\mathrm{C}^{18} \mathrm{O}$ column density at the target position; (4) average temperature of the central clump (envelope); (5) average $\mathrm{C}^{18} \mathrm{O}$ density of the central clump; (6) radius of the detected central clump; (7) the mass of the central clump; (8) total mass measured inside the primary beam (45.8"); (4) appearance of the silicate feature (Green et al. 2006; Larsson et al. 2000).

on the used envelope temperatures. For most of our target regions the excitation temperatures derived from the optically thick ${ }^{13} \mathrm{CO}$ emission are consistent with the $20-50 \mathrm{~K}$ temperatures used by studies of YSO envelopes (Andre \& Montmerle 1994; Hogerheijde et al. 1999; Sandell \& Weintraub 2001; Lommen et al. 2008; Dunham et al. 2012; Green et al. 2013; Kóspál et al. 2013). The APEX study by Kóspál et al. (2017) derived lower temperatures $(5-7 \mathrm{~K})$ for lower-mass envelopes and higher temperatures $(>50 \mathrm{~K})$ for high-mass envelopes. If we do not consider V2493 Cyg, V1735 Cyg with the highest envelope mass shows the highest average temperature $(25 \mathrm{~K})$ but the lower-mass envelope of V2492 Cyg is around this value as well. Somewhat higher temperatures are measured around the binary FUor, RNO 1B/C $(30-50 \mathrm{~K})$. One explanation for such high values could be the high-energy input from several strong outflows driven by young sources in the region.

The different evolutionary stages of YSOs can be distinguished by investigating their SED in the sub-millimetre and IR, which is also connected to the amount of circumstellar material associated with the central star. According to the dust continuum studies of YSOs in $\rho$ Ophiuchi and Taurus by Andre \& Montmerle (1994), Bontemps et al. (1996) and Moriarty-Schieven et al. (1994), envelope masses higher than $0.5 M_{\odot}$ are characteristic for Class 0 protostars that are still in their main accretion phase. Class I objects were found with masses between 0.015 and $0.15 M_{\odot}$ with a median of $0.06 M_{\odot}$ and Class II objects have masses of $0.002-0.15 M_{\odot}$ with a median of $0.01 M_{\odot}$. While the majority of Class III sources were undetected at $1.3 \mathrm{~mm}$, the circumstellar material around Class II stars remained usually unresolved with the approximately $1000 \mathrm{AU}\left(12^{\prime \prime}\right)$ resolution of the IRAM $30 \mathrm{~m}$ telescope. Additionally, Class I objects were detected mostly as extended, resolved sources with radii of less than a few thousand AU. Apart from the dust emission from the disk, part of the emission from the envelope is also included in the continuum detections of Class I sources (Terebey et al. 1993).

The line-based masses of the detected FUor envelopes presented in this paper span two orders of magnitude, from 0.024 to $0.51 M_{\odot}$. Similar masses were derived by Kóspál et al. (2017) using ${ }^{12} \mathrm{CO}$ and ${ }^{13} \mathrm{CO}$ measurements by APEX for eight southern and equatorial FUors. The continuum-based masses of the three strongly detected FUors (V1057 Cyg, V2492 Cyg, V1735 Cyg) are fairly uniform, around $0.3-0.4 M_{\odot}$. Some correlation of the dust masses with the line-based values can be seen for the three targets with no significant millimetre detections (V2493 Cyg, V733 Cep, V710 Cas), since they have also no or very small $\mathrm{CO}$ envelopes. The two targets with similar line-based masses (V1057 Cyg and V2492 Cyg) also have very similar continuum masses. However, V1735 Cyg with a high line-based envelope mass also shows a continuum mass similar to those two and 
V1515 Cyg, that was detected as a weak millimetre source, has the second highest line-based envelope mass. The differences in gas/dust can be explained by the difference in the mass of their circumstellar disk, the differing viewing angle of the system, and the different $\mathrm{CO}$ depletion or grain growth.

Considering the derived envelope masses, the evolutionary states of our FUor targets span both very young and embedded, early Class I stages (V1735 Cyg with a high-mass and extended gas envelope) and a more evolved, Class II stage (V2493 Cyg with no continuum detection and a smaller, low-mass envelope). The non-detection of gas features centred on V733 Cep and V710 Cas also suggests low-mass envelopes and they are weakly or not detected in the continuum, implying that they are also more evolved, similar to Class II objects. The envelope masses of V1057 Cyg, V1515 Cyg and V2492 Cyg implies a Class I classification but V1515 Cyg has a much more extended gas envelope and is only weakly detected at $2.7 \mathrm{~mm}$.

Gramajo et al. (2014) used the model set of Whitney et al. (2003b,a) and Robitaille et al. (2006) to model the SED of almost all known FUors and derived the parameters of the stars, the disks and the envelopes. They determined that generally FUors have higher mass disks and higher disk accretion rates than quiescent Class I and Class II sources in Taurus. Their best fitting models of our targets have outer envelope radii of a few thousands of AU, usually two times the values we calculated. For V2492 Cyg both the envelope radii (2000 AU) and the envelope mass $\left(0.2 M_{\odot}\right)$ from their model are very close to our linebased values. Amongst our targets they find the lowest envelope mass for V2493 Cyg $\left(0.06 M_{\odot}\right)$ and the highest for V1735 Cyg $\left(0.9 M_{\odot}\right)$ which agrees with our results as well. However, they derive almost a magnitude smaller masses for the envelopes of V1057 Cyg and V1515 Cyg. There is no clear correlation between their derived disk masses and the detectability of our targets in the $2.7 \mathrm{~mm}$ continuum. They classified V1515 Cyg, RNO 1B/C and V1735 Cyg as Class II sources and V1057 Cyg, V2492 Cyg and V2493 Cyg as Class I sources. They performed no modelling for V733 Cep.

Based on the appearance of the $10 \mu \mathrm{m}$ silicate feature Quanz et al. (2007a) defined two categories specifically amongst FUors: Category 1 stars show the feature in absorption, which is explained with a dusty and icy envelope surrounding the object. Category 2 stars show the feature in emission that arises from the surface layer of the circumstellar accretion disk. There is also evidence of grain growth in the emission profiles of Category 2 stars. These two groups can be explained if we assume that Category 1 FUors are younger, more similar to embedded Class 0 or Class I objects, while Category 2 FUors are more evolved. The accretion disk is visible to the observer because much of the envelope has already dispersed or accreted onto the central star and a wide cavity has opened up. The explanation is in agreement with theoretical model predictions that expect FUor outbursts to be recurring and then, when the envelope is cleared up, the star to enter a more quiescent phase. This way FUors can be the link between Class I and Class II stars and FUor outbursts may play the key role in this transition.

From this paradigm it follows that Category 2 FUors must have less massive envelopes than Category 1 FUors. According to Spitzer and ISO data (Green et al. 2006; Larsson et al. 2000) the spectra of V710 Cas, V1735 Cyg and V733 Cep show the silicate feature in absorption, V1515 Cyg and V1057 Cyg show it in emission, while there is no good spectral resolution data of the $10 \mu \mathrm{m}$ band towards V2492 Cyg and V2493 Cyg. V1735 Cyg shows the feature in absorption and this star has the most massive envelope in our sample, while V1057 Cyg and V1515 Cyg have lower mass envelopes and show the silicate feature in emission. This appears to confirm the correlation between the two parameters, however, the two other Category 1 FUors (V733 Cep and V10 Cas) have no detected CO clumps associated with them (Table 4), thus seem to be more evolved systems. The results are also ambiguous since two FUors with low-mass envelopes have no $10 \mu \mathrm{m}$ spectra available. Correlating the two parameters this way might be challenging since while silicate emission or absorption originates from the disk (the inner few $\mathrm{AU}$ for a low-mass star) and is spatially unresolved, CO structures can be found on both smaller and larger scales around the objects. For individual objects the viewing angle of the equatorial plane of the object also has a large effect on the silicate spectral shape. Even if the envelope is already depleted and the object is more evolved, viewing it edge-on will result in the absorption of the silicate band. We note however that since our objects are generally visible in the optical regime it is probable that even if they are embedded we see them somewhat from above the disk plane, around the direction of their envelope cavity.

Interestingly there is also a correlation of $M_{\text {total }}$ with the $10 \mu \mathrm{m}$ spectral feature: the stars showing the silicate feature in emission have total masses of 1.25 and $3.1 M_{\odot}$ and those that show it in absorption have total masses above $4.5 M_{\odot}$. However, calculating the total mass inside the primary beam clearly includes many other sources and their potential envelopes as well (as it is the case with V710 Cas). This way $M_{\text {total }}$ provides information about the larger scale environment of the star which still has an effect on the detected spectral features. In the case of V733 Cep and V710 Cas, where the non-detection in the continuum and the lack of a central CO clump suggests that their envelope has already strongly depleted, the absorption feature might be created by the large scale structures around them. In the case of V1057 Cyg and perhaps V1515 Cyg, which seem like embedded, Class I sources, we might see the $10 \mu \mathrm{m}$ emission since we observe the stars from the direction of their circumstellar cavity.

\section{Summary and conclusions}

We present an interferometric ${ }^{13} \mathrm{CO}$ and $\mathrm{C}^{18} \mathrm{O}$ survey of northern FU Orionis-type stars. Strong ${ }^{13} \mathrm{CO}$ and $\mathrm{C}^{18} \mathrm{O}$ emission was detected around each of our targets in a few thousand AU spatial scale, tracing extended circumstellar clouds that show substructure. The smaller-scale distribution of the emission peaks at or close to the optical positions of the FUors and is generally composed of a few significant or many clumps with complicated kinematic structure. In most cases the ${ }^{13} \mathrm{CO}$ emission is optically thick and can be used to derive temperatures, while $\mathrm{C}^{18} \mathrm{O}$ is optically thin and so suitable to determine the densities and masses of the detected structures. The calculated temperatures are mostly in the expected range for envelopes around lowmass stars $(20-50 \mathrm{~K})$. The peak $\mathrm{C}^{18} \mathrm{O}$ column densities in the envelopes range from a few times $10^{15}$ to $10^{16} \mathrm{~cm}^{-2}$ which gives $\mathrm{H}_{2}$ column densities of $0.5-5 \times 10^{22} \mathrm{~cm}^{-2}$. The temperature distribution also implies the heating of the detected small-scale central clumps by the targeted FUors.

V1057 Cyg: we found evidence of a rotating spherical clump centred on the star with a mass of $0.21 M_{\odot}$, that is also detected in the $2.7 \mathrm{~mm}$ continuum, implying a fairly large and dense envelope. The star was classified as a Class II object by its flat SED (Green et al. 2013) but its envelope mass and size suggests a younger, Class I object. Viewing the source from the direction of a large polar cavity that was previously proposed by Green et al. (2006) could explain the silicate emission feature and the SED. 
V1515 Cyg: we derive a similar mass for the envelope as for V1057 Cyg, and the silicate feature also appears in emission, which again, could be explained by viewing the source from the direction of its polar hole (Goodrich 1987). The SED is flat, suggesting a Class II object, and the continuum mass of the source is very low, which might also imply a somewhat more evolved state or an especially low-mass circumstellar disk. We find the lowest envelope temperature here in our sample. A roughly circular, ring-like, clumpy structure was detected around the FUor on the ${ }^{13} \mathrm{CO}$ channel maps that might trace the walls of an expanding shell, with a radius of $\approx 5000 \mathrm{AU}$.

V2492 Cyg: the envelope mass is very similar to V1057 Cyg and it is also detected at $2.7 \mathrm{~mm}$, suggesting a younger, embedded, Class I object. A large-scale velocity gradient was observed around the FUor, that may originate from the asymmetric compression of the material in the elongated, trunk-like cloud by the ionisation front generated by close-by luminous stars. We found many clumps behind the ionisation front, differing in density and mass, but many of them are clearly heated.

V2493 Cyg: an extended, clumpy cloud structure with high temperatures was observed around the target. We measured the lowest envelope mass and the smallest central CO clump here in our sample, the derived mass is close to the median envelope mass of Class II stars. The non-detection of the source in SMA $1.3 \mathrm{~mm}$ (Dunham et al. 2012) and in our $2.7 \mathrm{~mm}$ maps also suggests that it is more evolved.

V1735 Cyg: while the SED implies a Class II object, we found a massive, warm envelope that is detected at $2.7 \mathrm{~mm}$ as well, more characteristic of younger Class I stars. Perpendicular to a flattened, elliptical extended cloud structure, clear traces of a ${ }^{13} \mathrm{CO}$ outflow were found. The silicate feature appears in absorption which also implies an earlier evolutionary state.

V733 Cep: the environment around V733 Cep consists of two clumps on an offset from the position of the star. We found no $\mathrm{CO}$ or continuum emission that is clearly centred on the star, implying a later evolutionary state, but the silicate feature is seen in absorption. This might suggest the effect of the larger-scale circumstellar material on the spectral features. The FUor or another undetected object might also drive an outflow in the region.

$R N O 1 B / C$ : the envelope around the target was not detected but many clumps around the binary FUor system were. Some of them appear in the continuum as well, while the target was not detected at millimetre wavelength. The brightest detected CO clump with a mass of $1.8 M_{\odot}$ may be a circumbinary structure around $\mathrm{RNO} 1 \mathrm{~B}$ and $\mathrm{RNO} 1 \mathrm{C}$, and the reason behind the observed silicate absorption feature.

The classification of our objects into the FUor categories established by MIR observations of the silicate feature faces many problems. The determination of the exact evolutionary state is complicated by the unknown viewing angle of the sources, other embedded or young objects in the region and the lack of evenhigher-resolution spectral mapping around. However, we find that some of our targets exhibit parameters similar to very young, embedded Class I stars and some of them seem more evolved with only small envelopes, resembling early or late Class II YSOs. This diversity seems to reinforce the theory that FUors are Class I and Class II young stellar sources in a special evolutionary phase with bright eruptions and that these infall-driven FUor eruptions are the main driving force of this transition.

Acknowledgements. This research was supported by the Momentum grant of the MTA CSFK Lendület Disk Research Group. This work is based on observations carried out under project number VA6C with the IRAM Plateau de Bure Interferometer and under project number 260-11 with the IRAM 30 m Telescope. IRAM is supported by INSU/CNRS (France), MPG (Germany) and IGN (Spain). We are grateful to Michael Bremer for his help with the IRAM data reduction. The authors also wish to thank the anonymous referee for their valuable comments and suggestions.

\section{References}

Ábrahám, P., Juhász, A., Dullemond, C. P., et al. 2009, Nature, 459, 224 Andre, P., \& Montmerle, T. 1994, ApJ, 420, 837

Anglada, G., Rodriguez, L. F., Girart, J. M., Estalella, R., \& Torrelles, J. M. 1994, ApJ, 420, L91

Armitage, P. J., Livio, M., \& Pringle, J. E. 2001, MNRAS, 324, 705

Audard, M., Ábrahám, P., Dunham, M. M., et al. 2014, Protostars and Planets VI, 387

Bally, J., \& Reipurth, B. 2003, AJ, 126, 893

Bell, K. R., \& Lin, D. N. C. 1994, ApJ, 427, 987

Boley, A. C., Mejía, A. C., Durisen, R. H., et al. 2006, ApJ, 651, 517

Bonnell, I., \& Bastien, P. 1992, ApJ, 401, L31

Bontemps, S., Andre, P., Terebey, S., \& Cabrit, S. 1996, A\&A, 311, 858

Cieza, L. A., Casassus, S., Tobin, J., et al. 2016, Nature, 535, 258

Dobashi, K., Bernard, J.-P., Yonekura, Y., \& Fukui, Y. 1994, ApJS, 95, 419

Duncan, D. K., Harlan, E. A., \& Herbig, G. H. 1981, AJ, 86, 1520

Dunham, M. M., Arce, H. G., Bourke, T. L., et al. 2012, ApJ, 755, 157

Evans, II, N. J., Balkum, S., Levreault, R. M., Hartmann, L., \& Kenyon, S. 1994, ApJ, 424, 793

Goodrich, R. W. 1987, PASP, 99, 116

Gramajo, L. V., Rodón, J. A., \& Gómez, M. 2014, AJ, 147, 140

Green, J. D., Hartmann, L., Calvet, N., et al. 2006, ApJ, 648, 1099

Green, J. D., Evans, II, N. J., Kóspál, Á., et al. 2013, ApJ, 772, 117

Hales, A. S., Corder, S. A., Dent, W. R. D., et al. 2015, ApJ, 812, 134

Hartmann, L., \& Kenyon, S. J. 1996, ARA\&A, 34, 207

Herbig, G. H. 1977, ApJ, 217, 693

Hillenbrand, L. A., Miller, A. A., Covey, K. R., et al. 2013, AJ, 145, 59

Hogerheijde, M. R., van Dishoeck, E. F., Salverda, J. M., \& Blake, G. A. 1999, ApJ, 513, 350

Kóspál, Á. 2011, A\&A, 535, A125

Kóspál, Á., Ábrahám, P., Acosta-Pulido, J. A., et al. 2013, A\&A, 551, A62 Kóspál, Á., Ábrahám, P., Acosta-Pulido, J. A., et al. 2016, A\&A, 596, A52

Kóspál, Á., Ábrahám, P., Csengeri, T., et al. 2017, ApJ, 836, 226

Kun, M., Szegedi-Elek, E., Moór, A., et al. 2011, MNRAS, 413, 2689

Larsson, B., Liseau, R., Men'shchikov, A. B., et al. 2000, A\&A, 363, 253

Levreault, R. M. 1988, ApJS, 67, 283

Lommen, D., Jørgensen, J. K., van Dishoeck, E. F., \& Crapsi, A. 2008, A\&A, 481,141

McMullin, J. P., Waters, B., Schiebel, D., Young, W., \& Golap, K. 2007, in Astronomical Data Analysis Software and Systems XVI, eds. R. A. Shaw, F. Hill, \& D. J. Bell, ASP Conf. Ser., 376, 127

Momose, M., Ohashi, N., Kawabe, R., Nakano, T., \& Hayashi, M. 1998, ApJ, 504,314

Moriarty-Schieven, G. H., Wannier, P. G., Keene, J., \& Tamura, M. 1994, ApJ, 436, 800

Mosoni, L., Sipos, N., Ábrahám, P., et al. 2013, A\&A, 552, A62

Ossenkopf, V., \& Henning, T. 1994, A\&A, 291, 943

Quanz, S. P., Henning, T., Bouwman, J., Linz, H., \& Lahuis, F. 2007a, ApJ, 658, 487

Quanz, S. P., Henning, T., Bouwman, J., et al. 2007b, ApJ, 668, 359

Reipurth, B., \& Wamsteker, W. 1983, A\&A, 119, 14

Robitaille, T. P., Whitney, B. A., Indebetouw, R., Wood, K., \& Denzmore, P. 2006, ApJS, 167, 256

Ruíz-Rodríguez, D., Cieza, L. A., Williams, J. P., et al. 2017, MNRAS, 466, 3519 Sandell, G., \& Weintraub, D. A. 2001, ApJS, 134, 115

Scoville, N. Z., Sargent, A. I., Sanders, D. B., et al. 1986, ApJ, 303, 416

Staude, H. J., \& Neckel, T. 1991, A\&A, 244, L13

Terebey, S., Chandler, C. J., \& Andre, P. 1993, ApJ, 414, 759

Vorobyov, E. I., \& Basu, S. 2006, ApJ, 650, 956

Vorobyov, E. I., \& Basu, S. 2015, ApJ, 805, 115

Vorobyov, E. I., Zakhozhay, O. V., \& Dunham, M. M. 2013, MNRAS, 433, 3256

Weintraub, D. A., \& Kastner, J. 1993, ApJ, 411, 767

Whelan, E. T., Dougados, C., Perrin, M. D., et al. 2010, ApJ, 720, L119

Whitney, B. A., Wood, K., Bjorkman, J. E., \& Cohen, M. 2003a, ApJ, 598, 1079

Whitney, B. A., Wood, K., Bjorkman, J. E., \& Wolff, M. J. 2003b, ApJ, 591, 1049

Wilson, T. L. 1999, Rep. Progr. Phys., 62, 143

Yang, J., Ohashi, N., \& Fukui, Y. 1995, ApJ, 455, 175

Yonekura, Y., Dobashi, K., Mizuno, A., Ogawa, H., \& Fukui, Y. 1997, ApJS, 110,21

Zurlo, A., Cieza, L. A., Williams, J. P., et al. 2017, MNRAS, 465, 834 


\section{Appendix $A:{ }^{13} \mathrm{CO}$ and $\mathrm{C}^{18} \mathrm{O}$ channel maps}

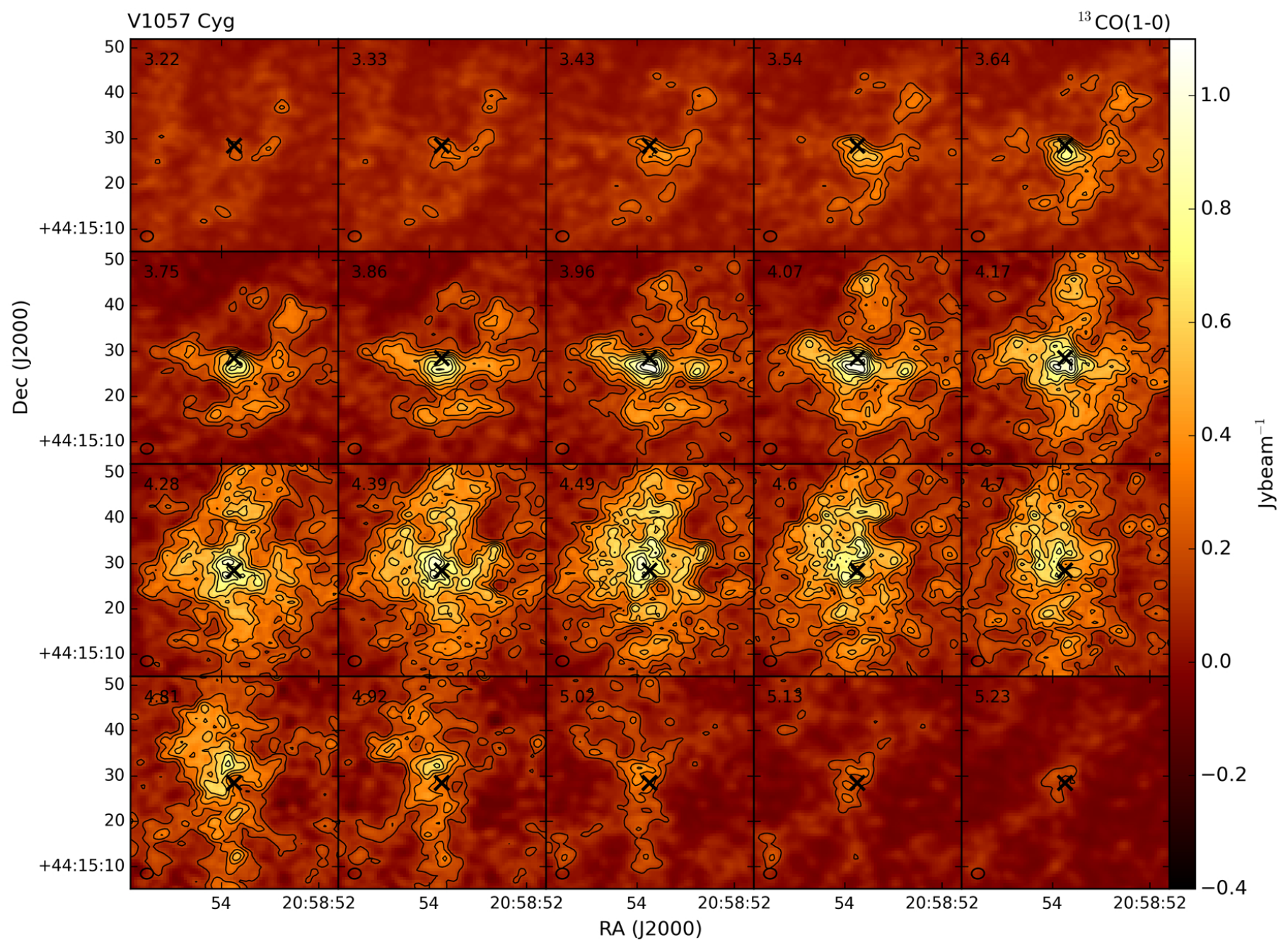

Fig. A.1. ${ }^{13} \mathrm{CO}$ channel maps of V1057 Cyg. The velocity of the channel is marked on the panels. The contours are at $10,15 \ldots \sigma$ $(\sigma=0.02) \mathrm{Jy}_{\text {beam }}{ }^{-1}$. 


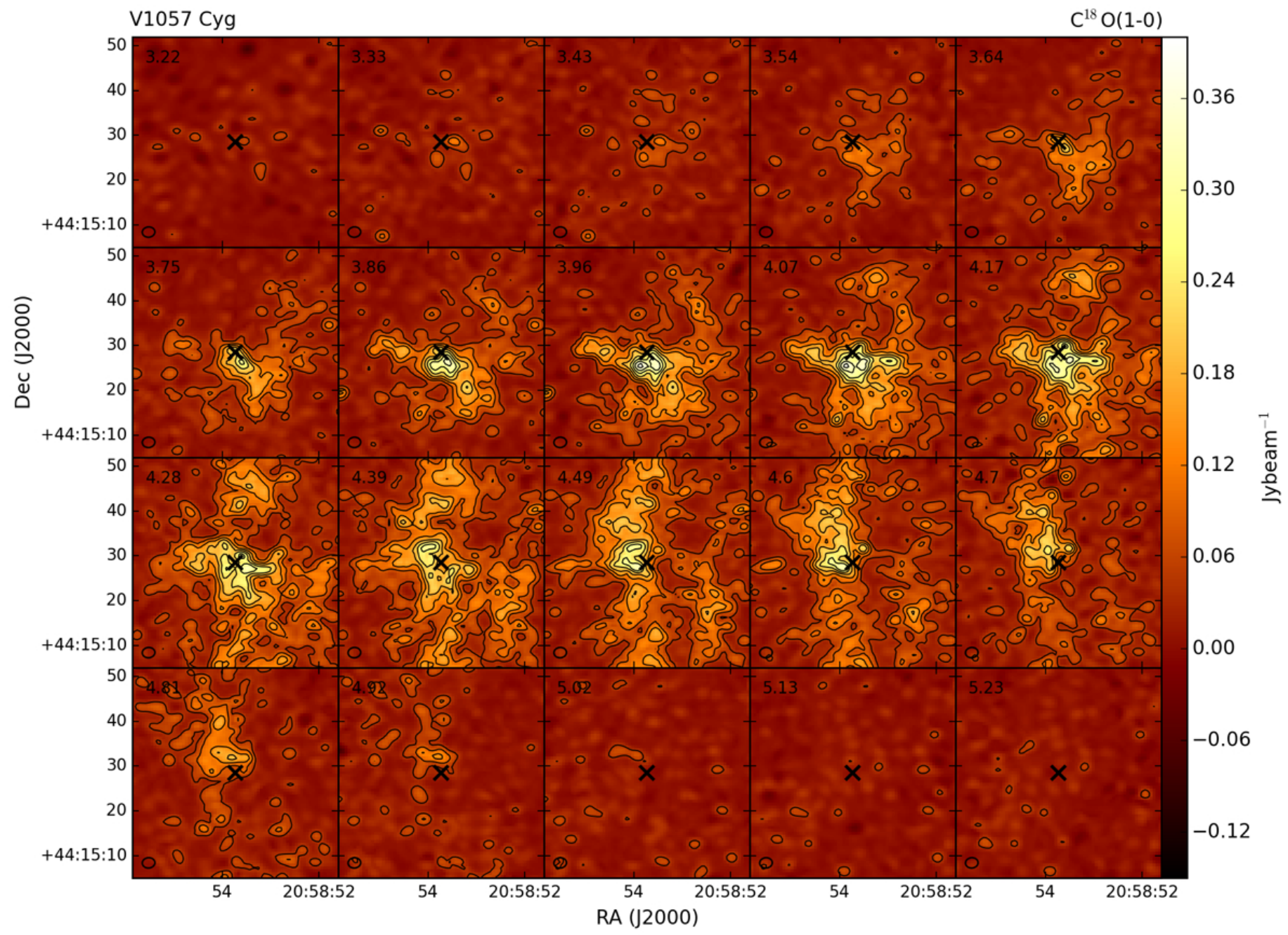

Fig. A.2. $\mathrm{C}^{18} \mathrm{O}$ channel maps of $\mathrm{V} 1057 \mathrm{Cyg}$. The velocity of the channel is marked on the panels. The contours are at $3,6, \ldots \sigma$ $(\sigma=0.015) \mathrm{Jy}_{\text {beam }}{ }^{-1}$. 
O. Fehér et al.: Interferometric view of the envelopes of northern FUors

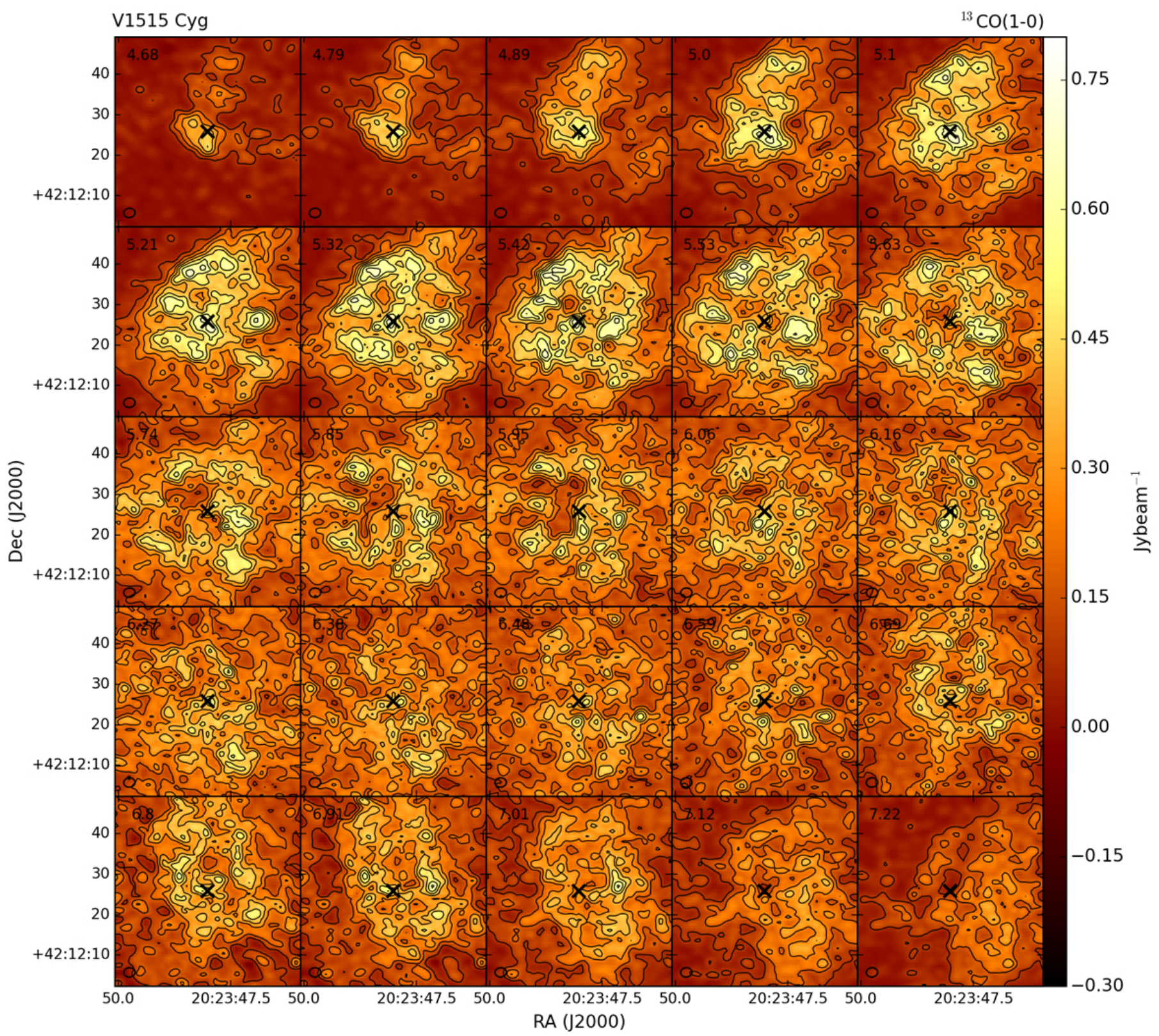

Fig. A.3. ${ }^{13} \mathrm{CO}$ channel maps of V1515 Cyg. The velocity of the channel is marked on the panels. The contours are at $3,6, \ldots \sigma(\sigma=0.03) \mathrm{Jy}$ beam ${ }^{-1}$. 


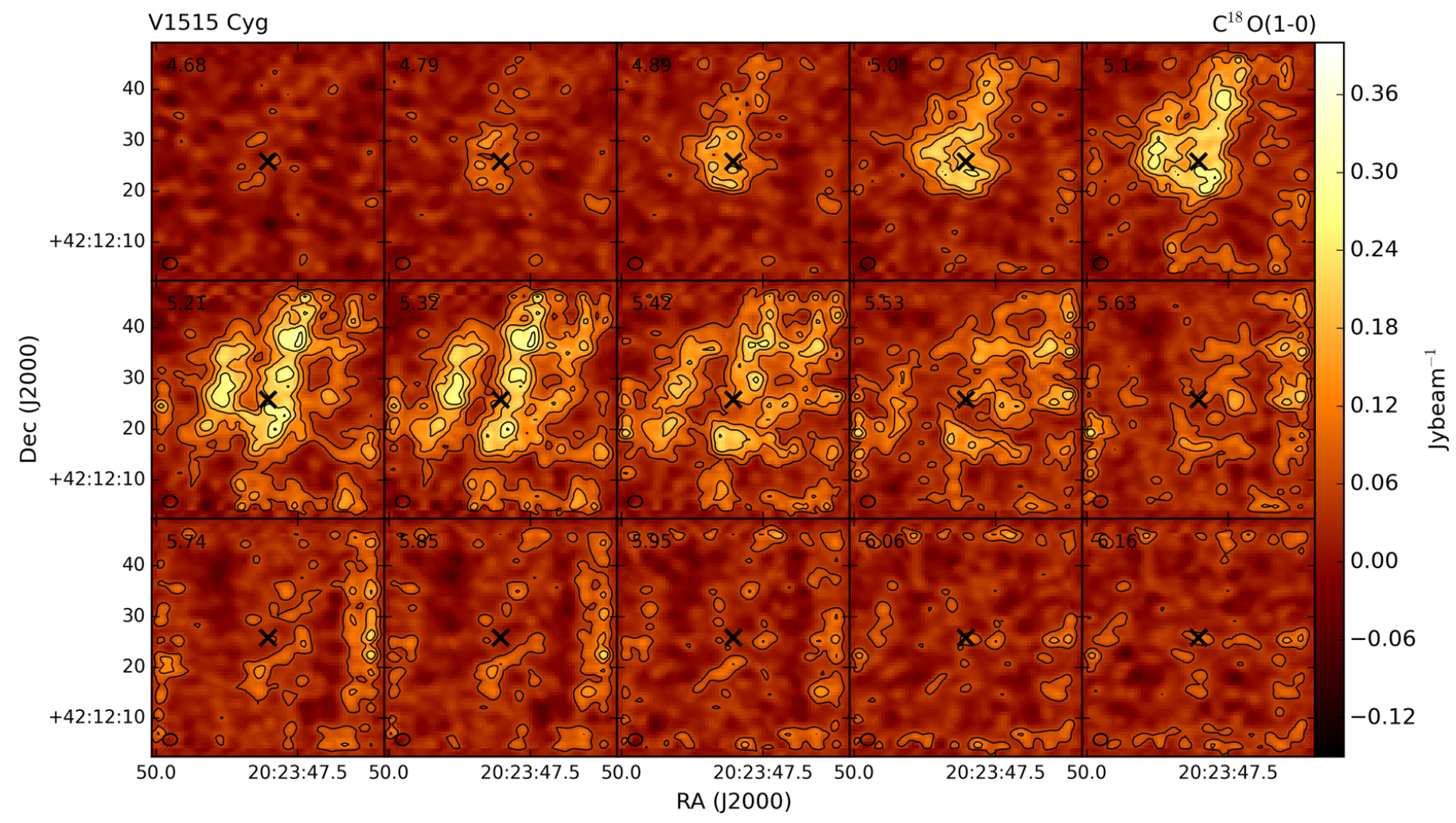

Fig. A.4. $\mathrm{C}^{18} \mathrm{O}$ channel maps of V1515 Cyg. The velocity of the channel is marked on the panels. The contours are at $3,6, \ldots \sigma(\sigma=0.02) \mathrm{Jy}$ beam ${ }^{-1}$. 
O. Fehér et al.: Interferometric view of the envelopes of northern FUors

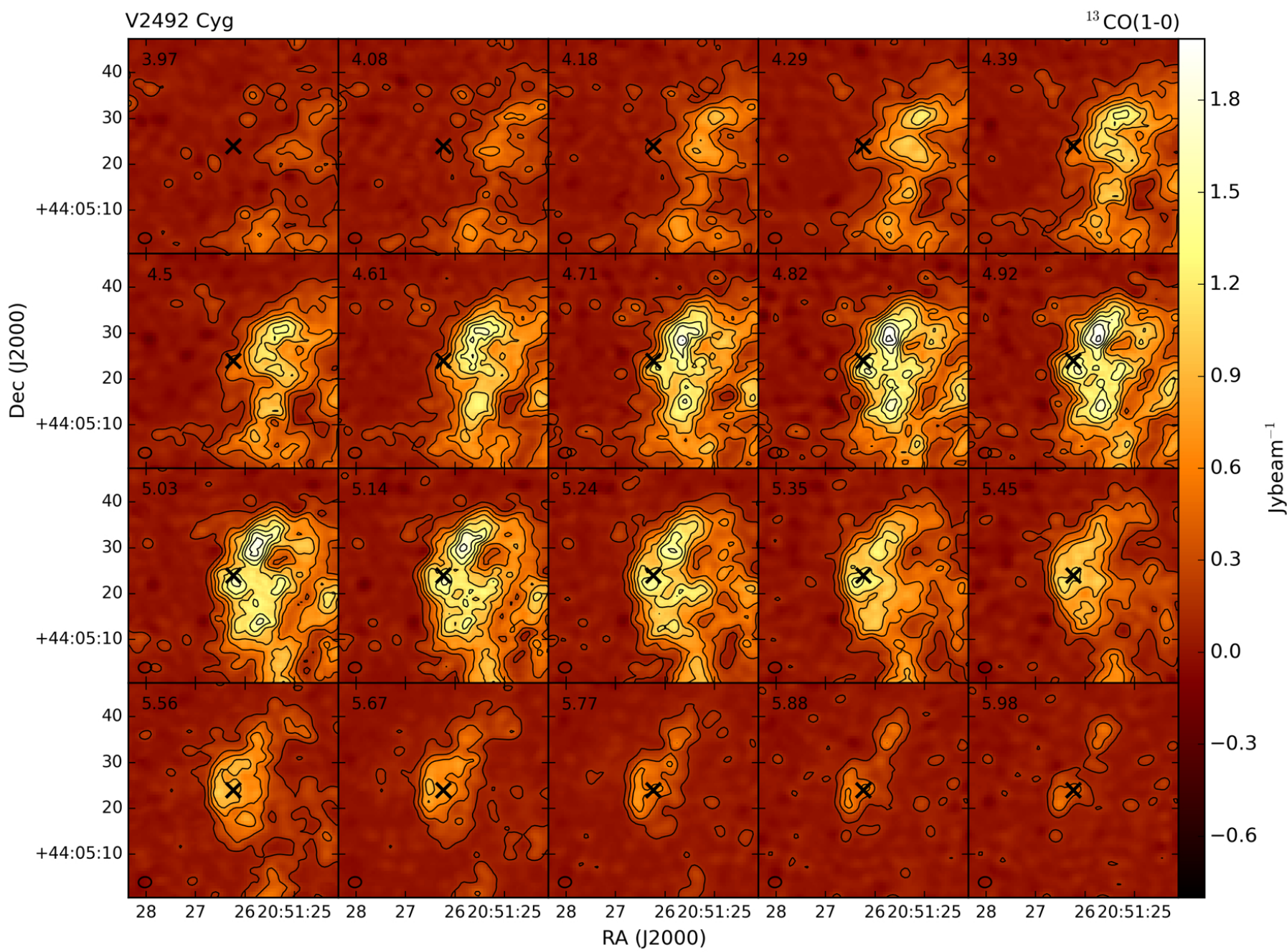

Fig. A.5. ${ }^{13} \mathrm{CO}$ channel maps of V2492 Cyg. The velocity of the channel is marked on the panels. The contours are at $3,9, \ldots \sigma(\sigma=0.04) \mathrm{Jy}$ beam ${ }^{-1}$. 


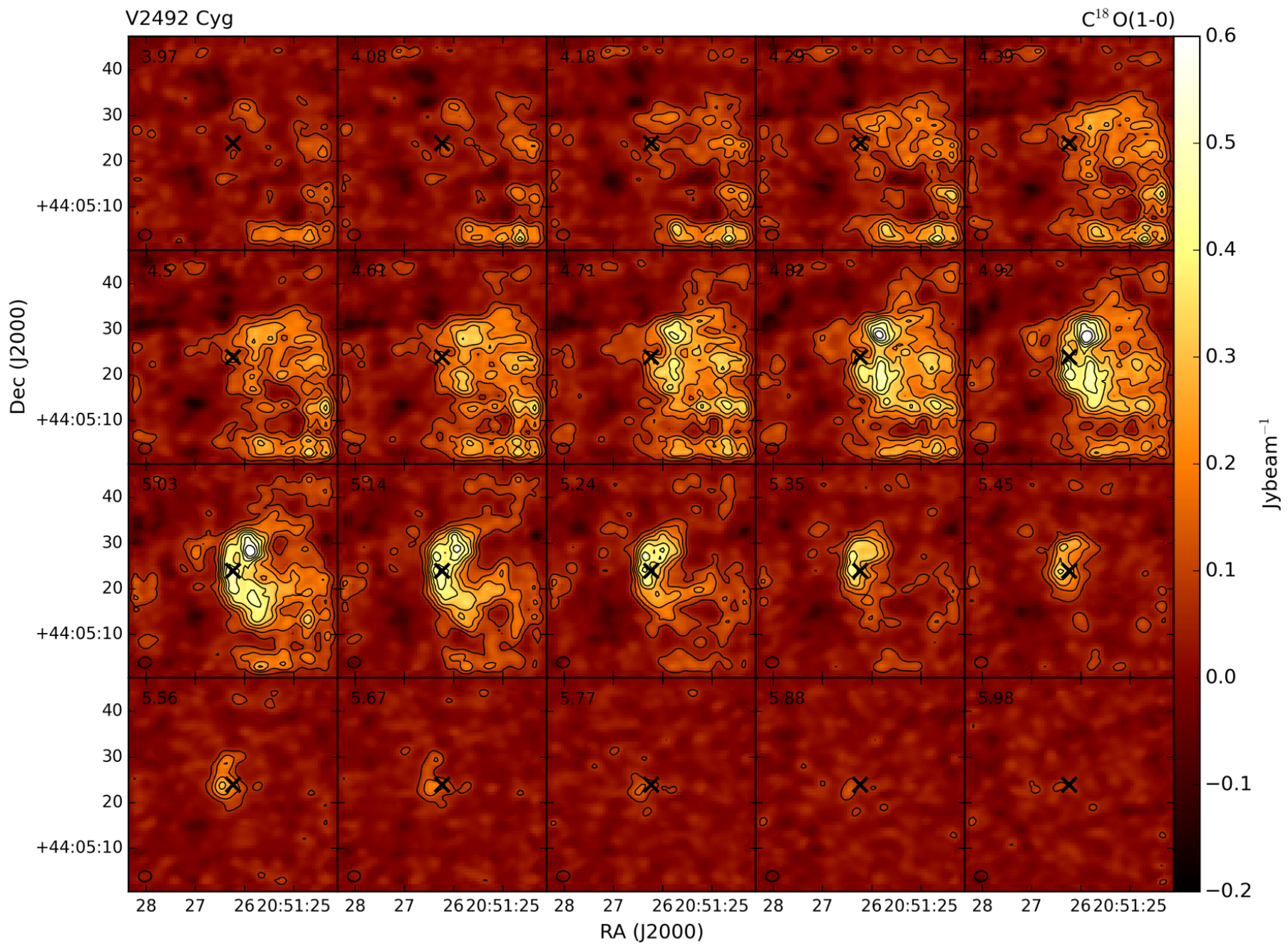

Fig. A.6. $\mathrm{C}^{18} \mathrm{O}$ channel maps of V2492 Cyg. The velocity of the channel is marked on the panels. The contours are at $3,6, \ldots \sigma(\sigma=0.24) \mathrm{Jy}$ beam ${ }^{-1}$. 
O. Fehér et al.: Interferometric view of the envelopes of northern FUors

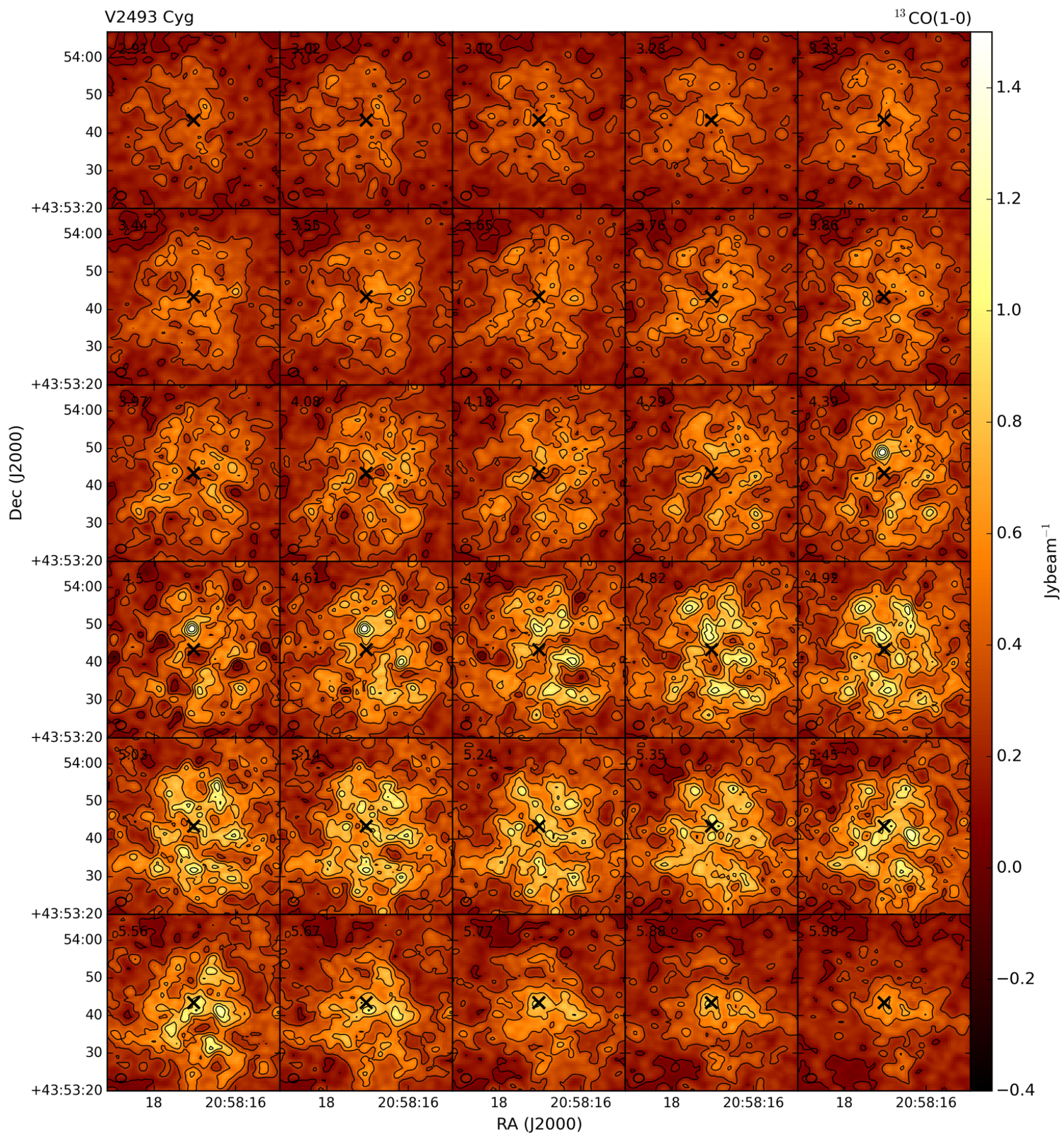

Fig. A.7. ${ }^{13} \mathrm{CO}$ channel maps of V2493 Cyg. The velocity of the channel is marked on the panels. The contours are at $3,9, \ldots \sigma$ $(\sigma=0.032) \mathrm{Jy} \mathrm{beam}^{-1}$. 


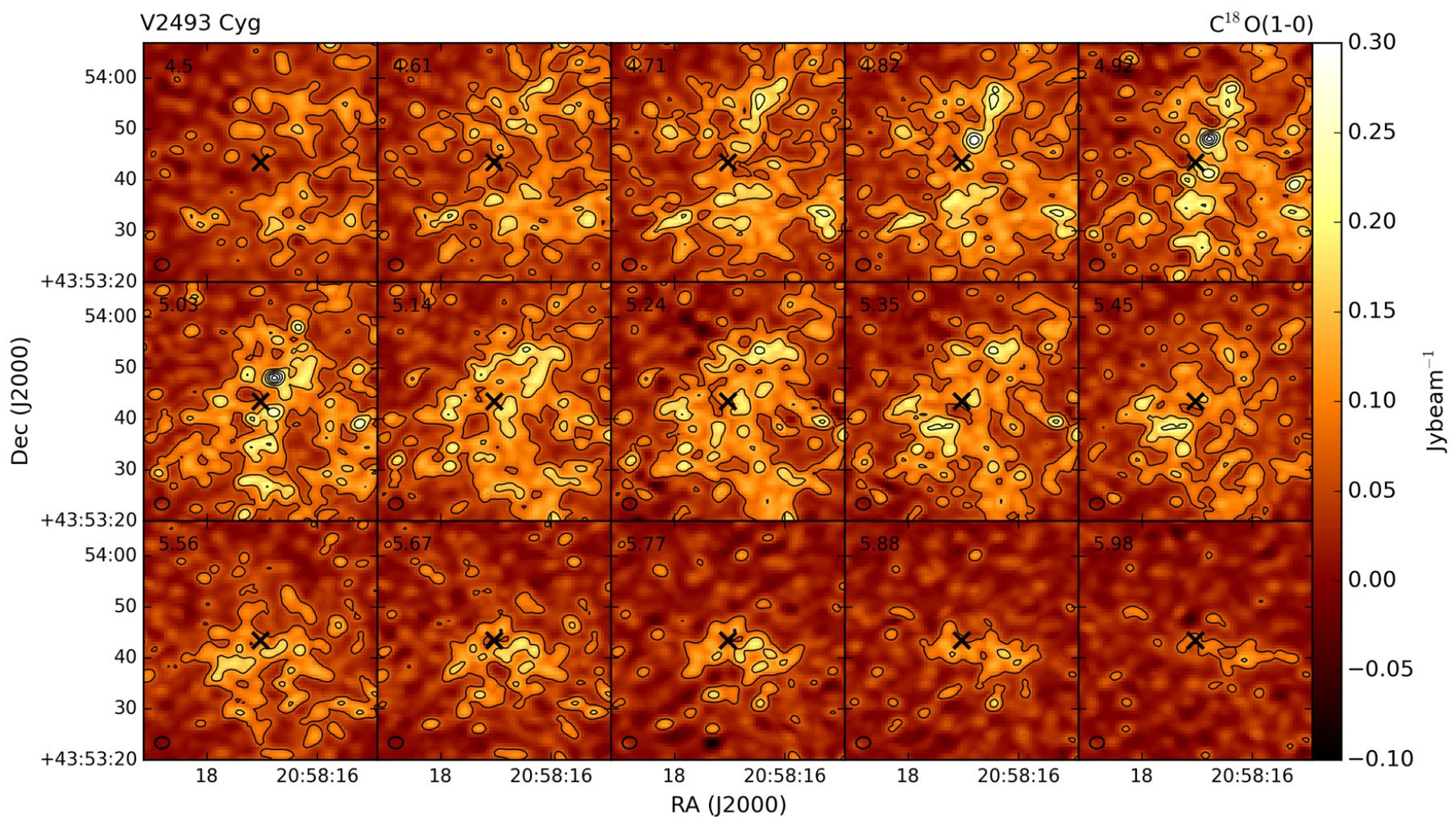

Fig. A.8. $\mathrm{C}^{18} \mathrm{O}$ channel maps of $\mathrm{V} 2493 \mathrm{Cyg}$. The velocity of the channel is marked on the panels. The contours are at $3,6, \ldots, \sigma$ $(\sigma=0.024) \mathrm{Jy} \mathrm{beam}^{-1}$. 
O. Fehér et al.: Interferometric view of the envelopes of northern FUors

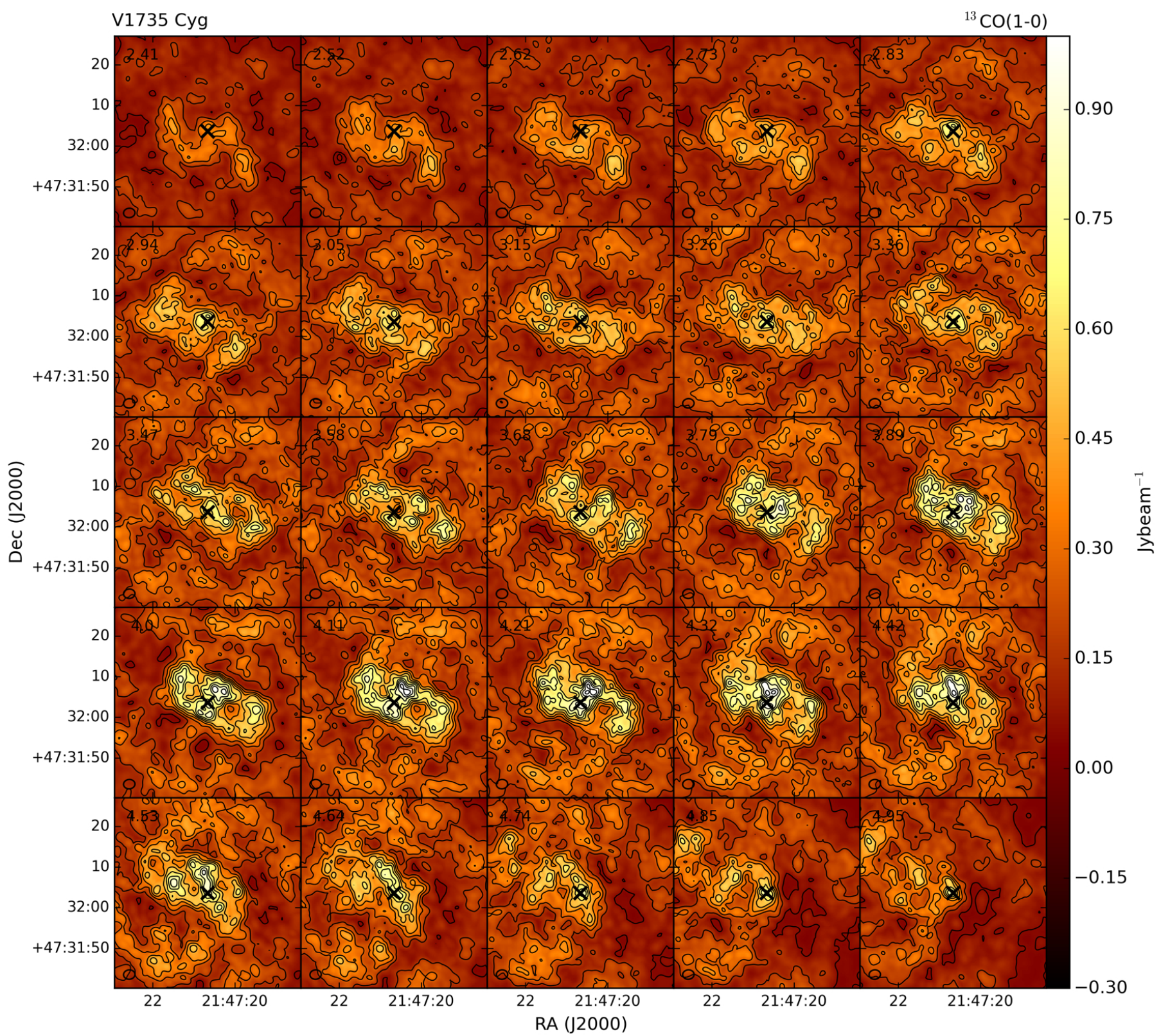

Fig. A.9. ${ }^{13} \mathrm{CO}$ channel maps of $\mathrm{V} 1735 \mathrm{Cyg}$. The velocity of the channel is marked on the panels. The contours are at $3,9, \ldots \sigma$ $(\sigma=0.018) \mathrm{Jy} \mathrm{beam}^{-1}$. 


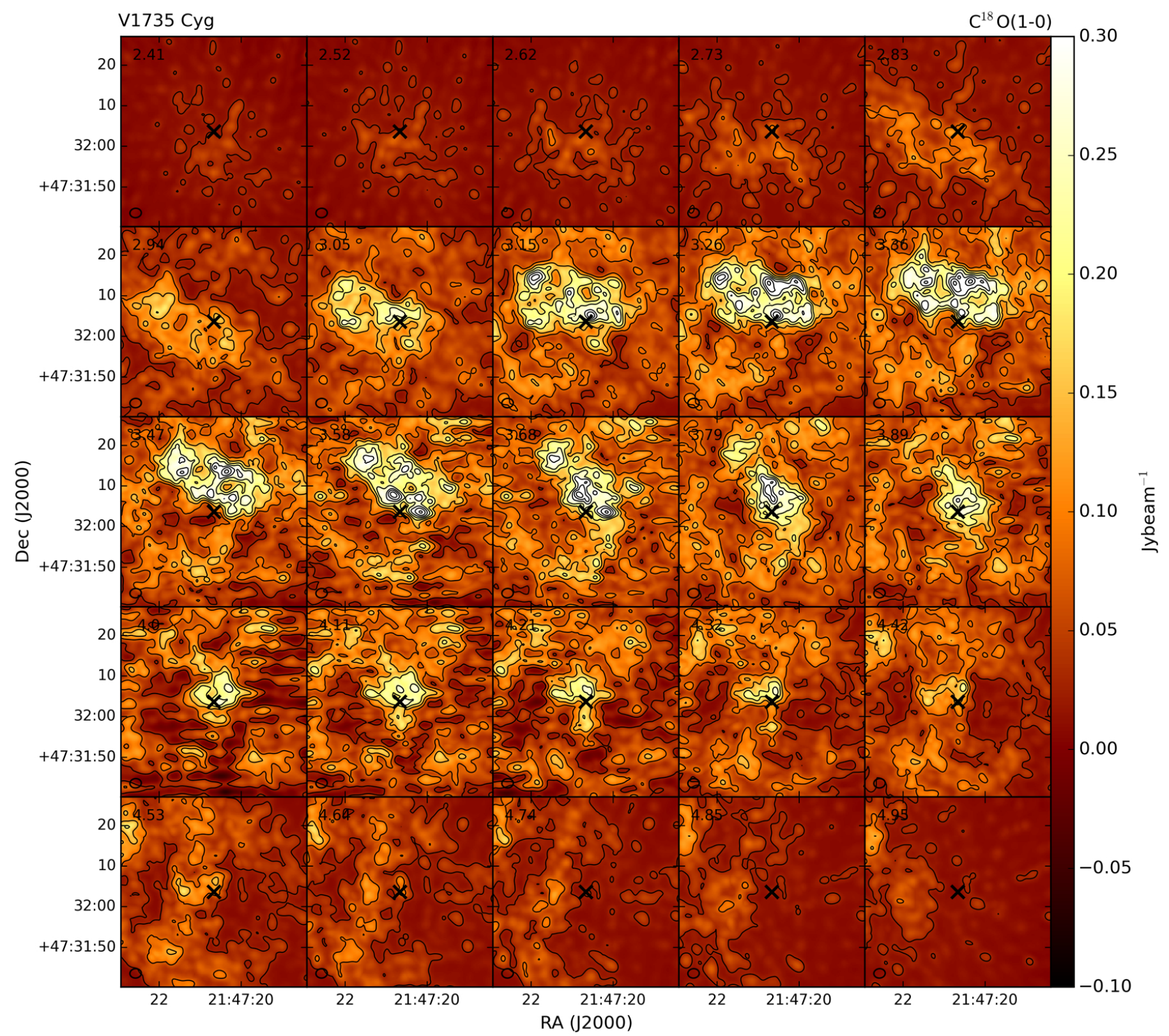

Fig. A.10. $\mathrm{C}^{18} \mathrm{O}$ channel maps of V1735Cyg. The velocity of the channel is marked on the panels. The contours are at $3,9, \ldots \sigma$ $(\sigma=0.009) \mathrm{Jy} \mathrm{beam}^{-1}$. 
O. Fehér et al.: Interferometric view of the envelopes of northern FUors

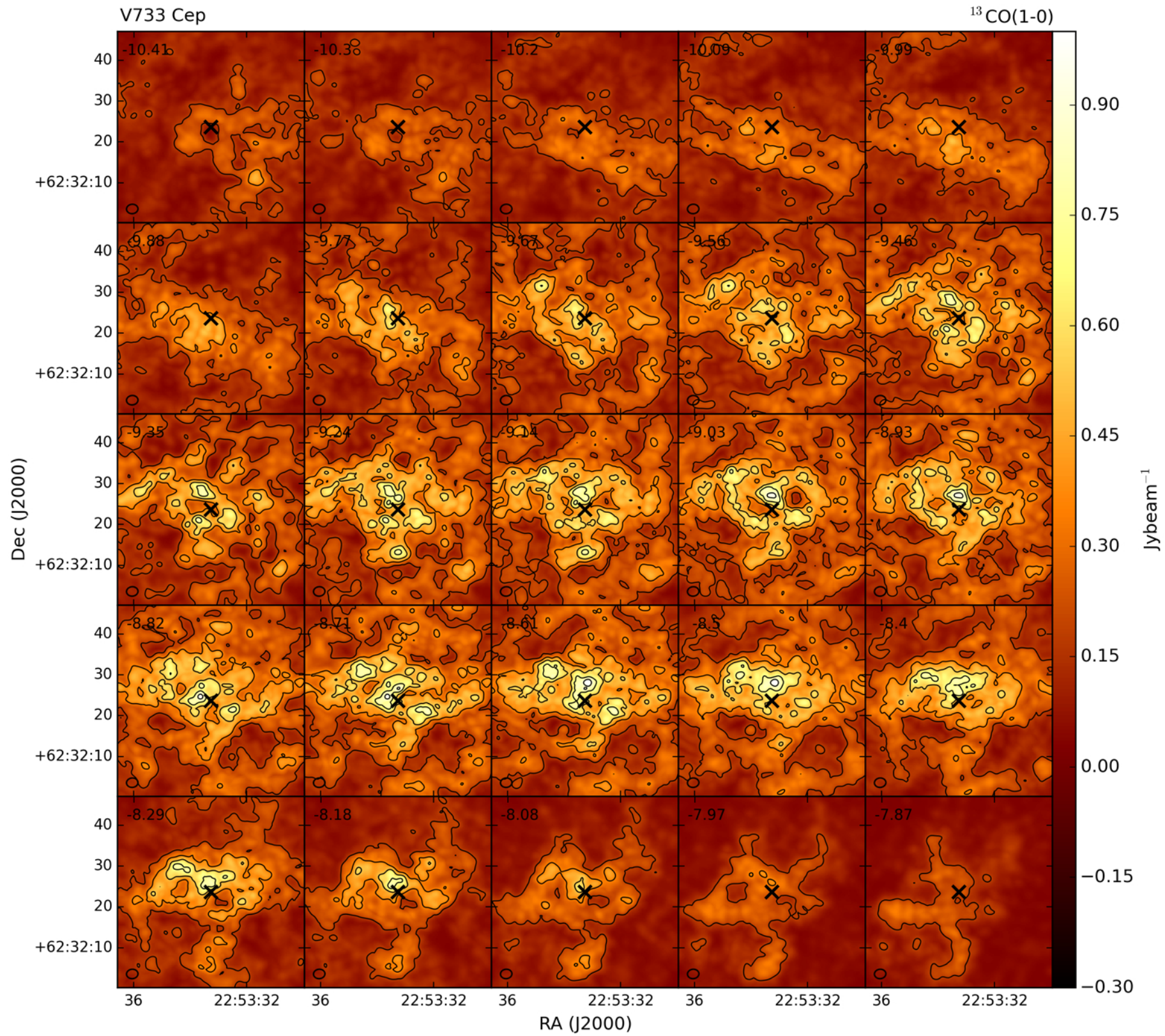

Fig. A.11. ${ }^{13} \mathrm{CO}$ channel maps of V733 Cep. The velocity of the channel is marked on the panels. The contours are at $10,20, \ldots \sigma$ $(\sigma=0.018) \mathrm{Jy} \mathrm{beam}^{-1}$. 


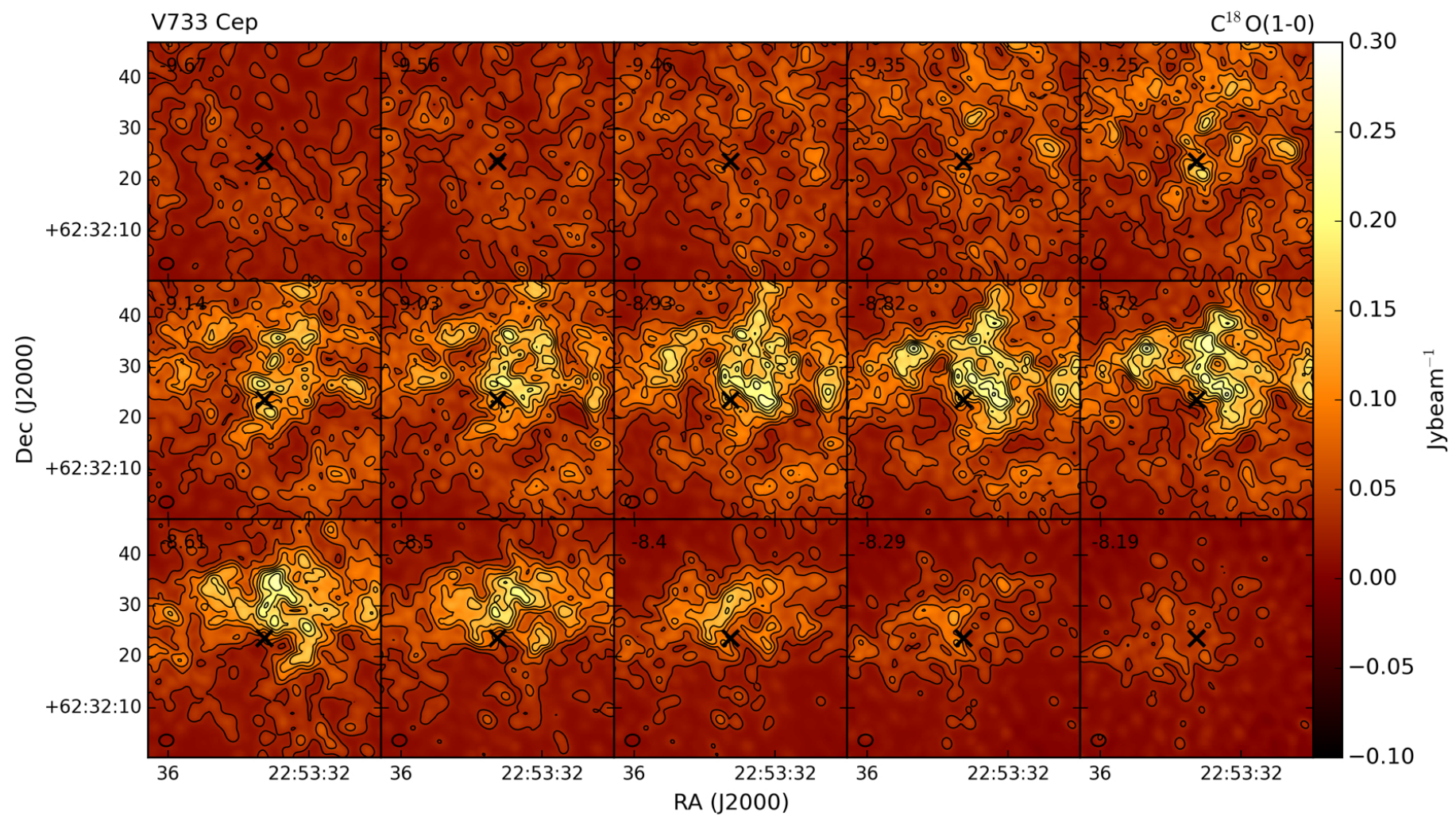

Fig. A.12. $\mathrm{C}^{18} \mathrm{O}$ channel maps of $\mathrm{V} 733 \mathrm{Cep}$. The velocity of the channel is marked on the panels. The contours are at $3,6, \ldots \sigma$ $(\sigma=0.009) \mathrm{Jy}_{\text {beam }}^{-1}$. 
O. Fehér et al.: Interferometric view of the envelopes of northern FUors

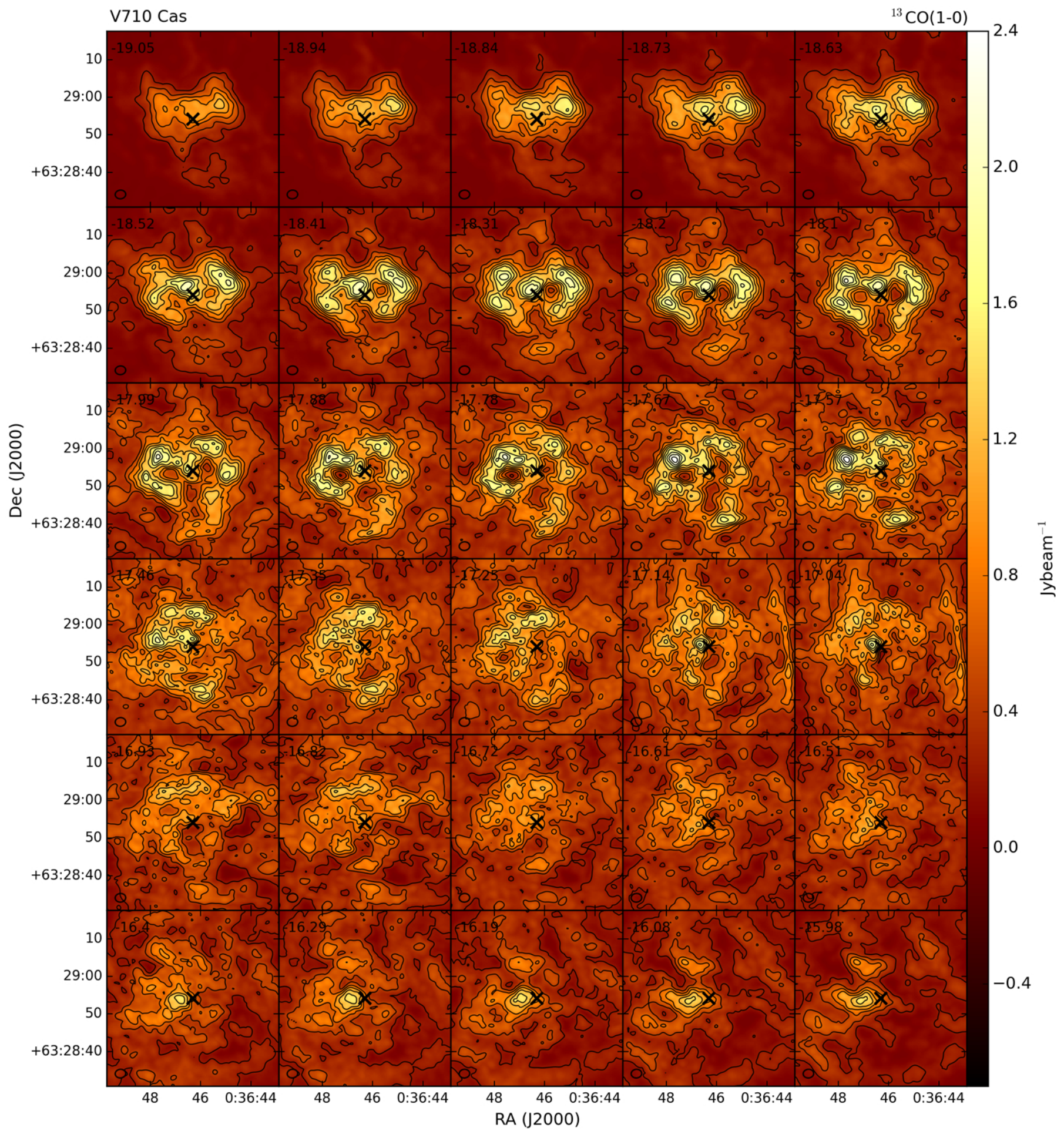

Fig. A.13. ${ }^{13} \mathrm{CO}$ channel maps of V710 Cas. The velocity of the channel is marked on the panels. The contours are at $10,20, \ldots \sigma$ $(\sigma=0.023) \mathrm{Jy}_{\text {beam }}^{-1}$. 


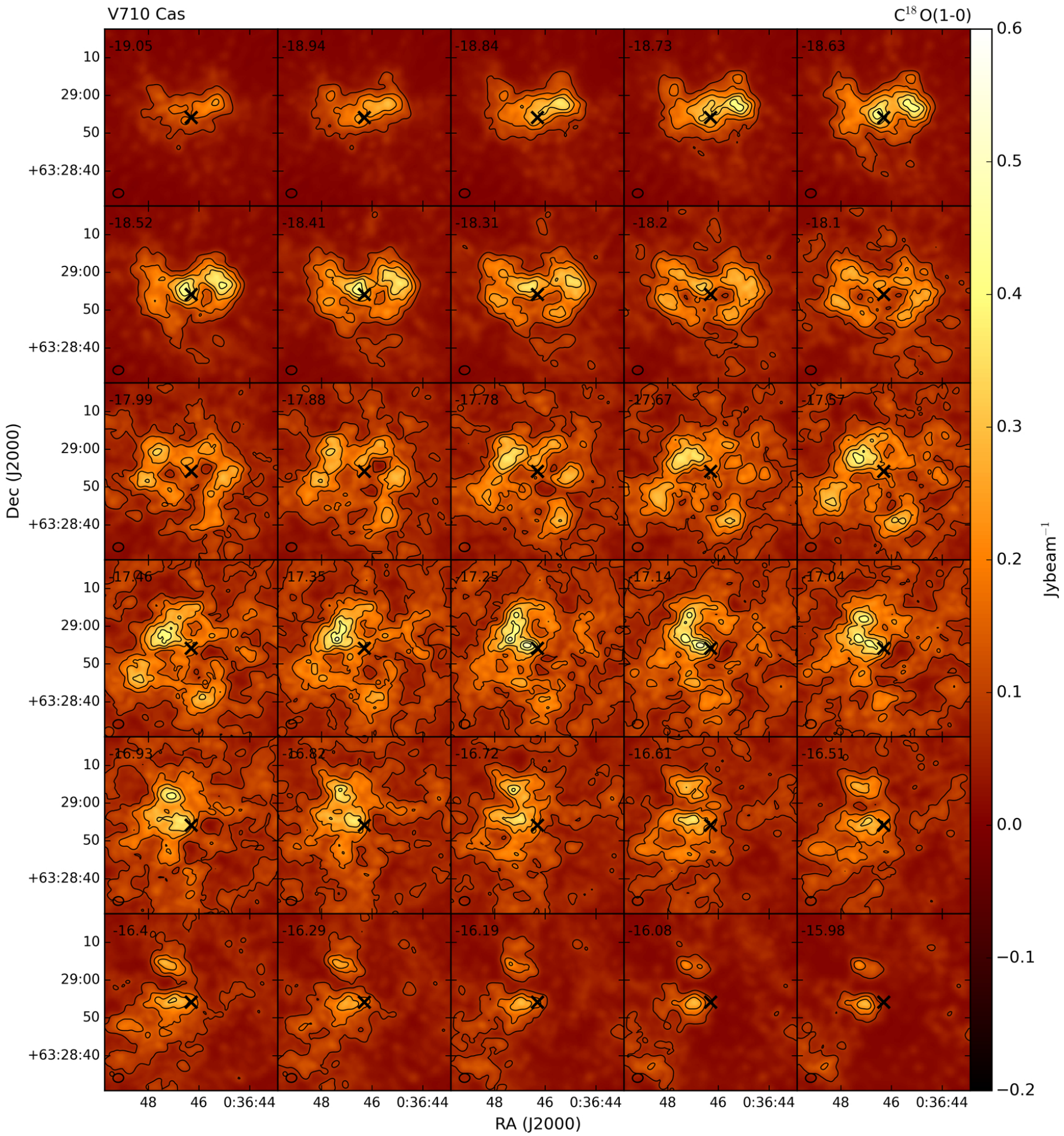

Fig. A.14. $\mathrm{C}^{18} \mathrm{O}$ channel maps of V710 Cas. The velocity of the channel is marked on the panels. The contours are at $10,20, \ldots \sigma$ $(\sigma=0.0076) \mathrm{Jy} \mathrm{beam}^{-1}$. 